\title{
Spectralanalytischer Nachweis von Spuren eines neuen, der eilften Reihe der Mendelejeff'schen Tafel angehörigen Elementes, welches besonders im Tellur und Antimon, ausserdem aber auch im Kupfer vorkommt
}

\author{
von \\ Dr. Anton Grünwald, \\ o. o. Professor der Mathematit an der k. K. deutschen techischen Hochsehule in Prag.
}

(Vorgelegt in der Sitzung am 10. October 1889.)

I.

Vor etwa zwei Jahren wurde ich von Professor W. N. Hartley in Dublin auf eine Reihe von sehr nahen Übereinstimmungen zwischen Wellenlängen verschiedener Elemente im Ultraviolett aufmerksam gemacht, welche er in seinen "Mensurements of the wavelengths of lines of high refrangibility in the spectra of elementary substances" Philos. Transact. London. Part I. 1884, p. 134 a. 135 in zwei Tafeln zusammengestellt hatte. Er bemerkte jedoch dabei zugleich, dass er unter mehr als 3000 Linien nur sehr wenige Coïncidenzen gefunden habe.

Da ich damals gerade durch andere Arbeiten ganz in Anspruch genommen war, konnte ich dieser Sache, so interessant sie auch war, nicht näher treten. Ich wurde jedoch in der neuesten Zeit bei Gelegenheit von Vergleicbungen verschiedener Spectra wiederholt auf mehrere sehr nahe Übereinstimmungen zwischen gewissen Wellenlängen, namentlich des Tellur-, Antimon-, und Kupfer-Spectrums geführt, fand aber dabei, dass ihre Zahl eine viel grössere war, als Hartley meinte. Ich erkannte auch bald den Grund davon. Hartley hatte, gleich Anderen, nur solche Linien verschiedener Spectra als wirklich mit einander ver- 
gleichbar angesehen, welche nicht nur in ihren Wellenlängen, sondern auch in ihrer äusseren Erscheinung übereinstimmten.

So richtig und nothwendig aber die letztere Anforderung bei der Identificirung von Linien eines und desselben Stoffes ist, welcher sich bei zrvei verschiedenen Beobachtungen und Messungen unter gleichen Unständen befunden hat, so unrichtig und anberechtigt ist sie bei der Vergleichung von Linien von übereinstimmenden Wellenlängen, welche demselben Stoffe unter verschiedenen Umständen gehören.

Angenommen, ein Element $X$ befinde sich in zwei verschiedenen Stoffen $\boldsymbol{A}$ und $\boldsymbol{B}$, welche Legirungen oder Gemische, ja selbst chemische Verbindungen der in ihnen enthaltenen Elemente sein können, wenn nur das Element $X$ in ihnen keine chemische Volumänderung (Condensation oder Dilatation) erfährt.

Dann wird das Element $X$ in beiden Stoffen zwar Strahlen von gleichen Wellenlängen aussenden; dieselben werden jedoch im Spectrum des Stoffes $A$ meistentbeils sowobl $a$ ) nach Intensität, als auch $b)$ nach anderen Merkmalen ihrer änsseren Erscheinung von den entsprechenden Strahlen im Spectrum des Stoffes $B$ verschieden sein.

a) Nach ibrer Intensität verschieden, weil die Amplituden der Atomtheilchen, welche im umgebenden Äther Strahlen von irgend einer Wellenlänge erzeugen, von anderen mit ihnen rhythmisch schwingenden Atomtheilchen der übrigen Componenten in dem Stoffe $\boldsymbol{A}$ and ers modificirt werden können, als im Stoffe $\boldsymbol{B}$.

(Bezüglich der "Atomtheilchen" siehe die Definition der chemischen Atome in meiner "Mathematischen Spectralanalyse des Magnesiums und des Kohlenstoffes" S. 5, 6 und beachte, dass die Bnzeichnung "Atomtheilchen" zwar einen philologischen, aber keinen realen Widerspruch in sich enthält, weil die chemischen Atome eben nur relativ, während der bekannten Processe, un getheilt bleibende Körperehen, nicht absolut untheilbare Wesen: ärcuot sind.)

b) Nach anderen Merkmalen ihrer Erscheinung verschieden; weil die Componenten, welche in $A$ und $B$ neben $X$ yorkommen, Linien erzeugen können, die rerschieden vertheilt, 
unnittelbar neben eine bestimmte Linie $\lambda$ des Elementes $X$ fallen, und der letzteren im Spectrum von $A$ ein Aussehen geben können, welches ron dem der gleich brechbaren Linie $\lambda$ desselben Elementes im Spectrum des Stoffes $\boldsymbol{B}$ gänzlich verschieden ist, so dass sie z. B. im Spectrum von $\boldsymbol{A}$ auf der Seite grösserer Brechbarkeit neblig erscheint, während sie im Spectrum von $B$ zu beiden Seiten von Nebel umgeben ist, oder mit den sie zunächst umgebenden Linien zu einem Bande verschwimmt etc. ${ }^{1}$

Würde man nun zwei derartige Strablen gleicher Brechbarkeit, deren Linien in den Spectren der Stoffe $\boldsymbol{A}$ und $\boldsymbol{B}$ verschiedene äussere Merkmale bezüglich ihrer Intensität und nächsten Umgebung aufweisen, nicht als zu demselben Elemente $X$ gehörig ansehen, und als nicht mit einander vergleichbar erklären, so würde man sich offenbar im Irrthume befinden, and sich selbst die Analyse und das Verständniss der Spectren der erwähnten Stoffe unmöglich machen.

„Bei der Vergleichung der Spectra verschiedener Stoffe $A$, $B$..., welche in der Absicht vorgenommen wird, etwaige Spuren gemeinsamer Elemente in denselben zu entdecken, sind nur die Well e n längen der Linien massgebend; die äussere Erscheinung der letzteren kommt erst bei der Untersuchung der Beziehungen zwischen den verschiedenen in den obigen Stoffen vorkommenden Elementen in Betracht."

Stellen wir uns jetzt vor, der Stoff $A$ enthalte eine gewisse Gewichtsmenge des Elementes $\boldsymbol{X}$. Diese werde nun allmälig vermindert, ohne die Gewichtsantheile der ubrigen Componenten von $A$, welche von $X$ chemisch unabhängig sind, zu ändern. Nur in dem Falle, wenn $X$ innerhalb $A$ auch in chemischen Verbindnngen mit anderen Elementen, etwa mit $Y$, von der Form $X Y$ vorkommen sollte, welche mit keiner chemischen Condensation von $X$ rerbunden sind, soll auch der Gewichtsantheil des jeweilig

1 Ja es ist sogar nicht ausgeschlossen, dass $\mathbf{k}$ |eine, bloss physikalische Variationen einer und derselben chemischen Condensation von $X$ in $A$ und $B$ kleine, von einander verschiedene Variationen der zu $X$ gehörigen Wellenlängen, und dadurch kleine Differenzen der letzteren von mehreven Hundertsteln bis zu wenigen Zehnteln einer Angströ m'schen Einheit in $A$ und $B$ erzeugen können. 
mit $X$ verbundenen Elementes $Y$ proportional zugleich mit $X$ vermindert werden, eine Voraussetzung, welche für den Fortbestand der Verbindungen von der Form $\boldsymbol{X Y}$ bei Verminderung des Gewichtsantheiles von $X$ nothwendig ist. Verbindungen des Elementes $X$ mit anderen, welche nur unter Condensation (also unter chemischer Volumänderung) der Componenten zu Stande kommen, sollen, wie bereits früher angenommen wurde, nicht in $A$ vorkommen. Die Gewichtsverminderung von $X$ soll unter den obigen Voranssetzungen so weit gehen, dass schliesslich nur mehr Spuren vou $X$ in dem so veränderten Stoffe $A$, welcher dann $A^{\prime}$ heissen möge, übrig bleiben.

Welchen Einfluss werden derartige Veränderungen auf das Spectrum von $\boldsymbol{A}$ haben? Oder mit andern Worten, welche Unterschiede werden zwischen den Spectren der Stoffe $\boldsymbol{A}$ und $\boldsymbol{A}^{\prime}$ zu erwarten sein?

Es ist bereits von den Professoren G. D. Liveing und J. Dewar in Cambridge, England, auf Grund einschlägiger Beobachtungen bemerkt worden, dass in solchen Fällen in der Regel alle sichtbaren Strahlen (überhaupt die Strahlen geringerer Brechbarkeit) rascher an Intensität bis zum Verschwinden abnehmen, als die Strahlen grosser Brechbarkeit, insbesondere die ultravioletten; so dass schliesslich, wenn schon alle sichtbaren Strahlen von $X$ im Spectrum des Stoffes $\boldsymbol{A}^{\prime}$ gan $z$ verschwunden sind, doch noch die ultravioletten, namentlich die brechbarsten) Strahlen von $X$ im Spectrum $A^{\prime}$ auf der photographischen Platte sichtbar und messbar sind, selbst dann, wenn sich in $A^{\prime}$ nur mehr eine so geringe Spur des Elementes $X$ befindet, dass sie durch directe chemische Analyse nur bei fabriksmässiger Aufarbeitung sehr grosser Mengen des Stoffes $A^{\prime}$ in w ägbarer Menge nachgewiesen werden könnte.

Meine Erklärung dieser Erscheinung ist sehr einfach. Bei gleicher Amplitude der Schwingungen werden die schnelleren Schwingungen der Strahlen grösserer Brechbarkeit, insbesondere der ultravioletten, eine grössere Energie besitzen, also auch unter sonst gleichen Umständen eine grössere Wirksamkeit auf die photographische Platte ausuiben, als die langsameren Schwingungen von Strahlen geringerer Brechbarkeit, wie die sichtbaren oder gar die infrarothen. 
Ein etwas abweichendes Verhalten können unter den angegebenen Verhältnissen einzelne minder brechbare Strahlen $\lambda$ des Elementes $X$ in dem Falle aufweisen, wenn die ibrigen Componenten, welche in $\boldsymbol{A}^{\prime}$ neben einer Spur von $\boldsymbol{X}$ rorkommen, Atomtheilchen enthalten, die mit grosser Intensität und rhythmisch mit dem Strahle $\lambda$ sehwingen. In einem solchen Falle $\mathrm{k} \ddot{o n} \mathrm{n}$ en die Atomtheilchen von $X$, welche im umgebenden $\ddot{A}$ ther den Strahl $\lambda$ erzengen, durch die rhythmisch verwandten Strahlen, welche die erwähnten Componenten dem Äther einprägen, eine solehe Verstärkung: ihrer Schwingungsamplitude erfahren, dass die von ihnen ausgehenden intensiveren Strahlen $\lambda$ mit der Abnahme der Gewichtsmenge von $X$ in $A$ nicht so rasch verblassen und schliesslich erlöschen, als es sonst der Fall wäre, und als es vielleicht bei benachbarten Strahlen von $X$, die eine solche Verstärkung wegen Mangel an ihnen rhythmisch verwandten Strahlungen der übrigen Componenten von $A$ nicht erfahren, wirklich der Fall ist.

Hieraus folgt, dass „fïr die Aufsuchung ron Spuren bekannter oder noch unbekannter Elemente $X$ im Spectrum eines Stoffes $A$ die Untersuchung des brechbarsten, insbesondere also des ultravioletten Theiles des Spectrums von A, eventuell seine Vergleichung mit anderen bekannten Spectren massgebend ist."

II.

Professor W. N. Hartley und W. E. Adeney haben in den bereits erwähnten "Mensurements of the wavelengths etc." Phil. Transact. London. Part. I. 1884 ihre Messungen der Wellenlängen der brechbarsten Strahlen der Luft, des $\mathrm{Mg}, \mathrm{Zn}, \mathrm{Cd}, \mathrm{Al}, \mathrm{Jn}, \mathrm{Tl}$, $\mathrm{Cu}, \mathrm{Ag}, \mathrm{C}, \mathrm{Sn}, \mathrm{Pb}$, Te. $\mathrm{As}, \mathrm{Sb}, \mathrm{Bi}$ und $\mathrm{Hg}$ mitgetheilt. Ihre $\mathrm{Me}-$ thode war im Wesentlichen die alte Cornu'sche (p. 66). Als Bezugslin ien wurden die wohldefinirten Linien einer KadmiumZinn-Legirung benützt; so dass die für die verschiedenen Metalle erhaltenen Wellenlängen un tere in ander vollkommen vergleichbar sind. (p. 88).

Wenn es daher nothwendig werden sollte (siehe weiter unten!), die von den Verfassern in ihren Tafeln gegebenen Wellenlängen zu rectificiren, so könnte dies mit Hülfe einer Interpolationscurve geschehen, welche aus den Angaben ibrer Mikrometer- 
seala und aus den zugehörigen verbesserten Wellenlängen abzuleiten wäre (p. 90).

Eine einfachere Methode, welche ich „die Methode der Fehlerdifferenzencurven" nennen will, führt jedoch in diesem und in ähnlichen Fällen leichter und rascher zu demselben Ziele.

Nach dieser Methode stellt man sich zunächst innerhalb des correcturbedürftigenWellenlängengebietes diefehlerhaftenWellenlängen $\lambda_{a}$ von möglichst vielen, nahe aneinanderliegenden Linien, nebst deren genaueren Werthen $\lambda_{w}$ zusammen, und bestimmt die relativen Fehler der ersteren gegen die letzteren: $\lambda_{w}-\lambda_{a}=\lambda_{a, w}$, welche offenbar die Differenzen ihrer unbekannten wabren Fehler sind. Die zu corrigirenden Wellenlängen $\lambda_{a}$ (z, B. Hartley's Zahlen) werden zu Abscissen, die relativen Fehler oder Abweichıngen $\lambda_{a, w}$ dagegen nach einem, im Vergleiche zu dem für die Abscissen gewählten, stark vergrösserten Massstabe zu Ordinaten genommen, auf Millimeterpapier die zu den gegebenen Coordinaten $\lambda_{a}, \lambda_{a, w}$ gehörigen Punkte construirt, und dieselben durch eine stetig gekrümmte Linie so verbunden, dass die Bögen zwischen zwei aufeinander folgenden Punkten möglichst wenig von gerallen Linien abweichen.

Will man nun für irgend eine (zwischen den ursprünglich ge geben en fehlerhaften liegende) Wellenlänge $\lambda_{a}$ des correcturbediurftigen Gebietes deren genaneren Werth ermitteln, so entnehme man ans der Zeichnnng die zur Abscisse $\lambda_{a}$ gehörigen Ordinate $\lambda_{a, v}$ und addire diese zu der gegebenen fehlerhaften Wellenlänge $\lambda_{a}$. Die so gefundene Zahl $\lambda_{a}+\lambda_{a, w}=\lambda_{w}$ ist dann die gesuchte richtigere Wellenlänge.

Ähnlich wie Hartley und Adeney haben auch die Professoren G. D. Liveing und J. Dewar die ultravioletten Spectren eiver Reihe von Elementen mit grosser Sorgfalt und Genauigkeit beobachtet, und die Ergebnisse ihrer Messungen in den Philos. Transact. London 1883. Vol. 174. Part I. „On the ultraviolet spectra of the elements" (Fe, Cu, K, Na, Li, Ba, Sr, Ca, $\mathbf{Z n}, \mathbf{A u}, \mathbf{T l}, \mathbf{A l}, \mathbf{P b}, \mathbf{S n}, \mathbf{S b}, \mathbf{B i}, \mathbf{C})$ und den Phil. Transact. 1888 ("Cobalt und Nickel") mitgetheilt.

Diese Messungen setzen uns nun in den Stand, die von Hartley und Adeney einerseits, von Liveing und Dewar anderseits bestimmten Wellenlängen identischer Linien in ver- 
s c hiedenen Spectren, (Cu, Zu, Tl, Al, Pb, Sn, Sb, Bi, C ....) mit einander zu vergleichen, deren relative Fehler(Abweichungen) zu ermitteln, und die Fehlerdifferenzencurve für den Übergang von den Hartley-Adeney'sehen zu den entsprechenden Liveing-Dew ar'schen Zahlen zu construiren.

Die Bestimmungen

1. der Wellenlängen des Kadmiums nach Rowland's Methode durch L. Bell in Baltimore ("The ultraviolet spectrum of cadmium “ American Journal of Science 1886, 3. series. Vol. 31. p. 426-431), von welchen neuestens mehrere auch von Liveing. und Dewar gemessen wurden (siehe deren obige Schrift über Kobalt und Nickel, p. 232, 233)

2. der Wellenlängen des Kupfers im Ultraviolet von $2369 \cdot 9$ bis $1944 \cdot 1$ Rowl. Scala, und deren Zusammenstellung mit entsprechenden Messungen von Liveing und Dewar, sowie von Hartley und Adeney durch J. Trowbridge und W. C. Sabine ("On wavelengths of metallic spectra in the ultraviolet" Proceed. of the American Academy, auch Philos. Magazine 1888, p. 342-35̃3), endlich

3. die werthvollen Messungen der Eisenlinien durch Kayser und Runge („Über die Spectra der Elemente." Berlin 1888) und deren Zusammenstellung mit entsprechenden Messungen von Liveing und Dewar, Cornu, Thalén and Vogel dürften schon jetzt die Reduction der nach der älteren Corn a'schen Methode ausgeführten Messungen mehrerer Beobachter auf die letzterwähnten nach der genaueren und verlässlicheren Rowland'schen Methode dnrchgeführten ermöglichen.

Von besonderem Interesse ist in dieser Beziehung die Construction der Fehlerdifferenzencurven für den Übergang von den Hartley-Adeney'schen und Liveing-Dewar'schen Zahlen zu den von $L$. Bell, Trowbridge und Sabine und von Kayser und $R$ unge angegebenen.

Die Vergleichung der Wellenlängen der sowohl von Liveing und Dewar, als auch von Hartley und Adeney gemessenen und mit Sicherheit identificirbaren Linien verschiedener Elemente lehrt, dass die Wellenlängen $\lambda$ ron Hartley und Adeney fürr $\lambda>2370$ gut mit den entsprechenden von Liveing und Dewar gegebenen stimmen. Die Abweichungen übersteigen selten $0 \cdot 4$. 
Für $\lambda \leqq 2370$ dagegen sind die Wellenlängen von Hartley und A deney mit starken Fehlern behaftet, welche hauptsächlich in der fehlerhaften Bestimmung der als Bezugslinien verwendeten Kadmiumlinien dieser Region ihren Grund haben. Ihre in verschiedenen Spectren innerhalb der genannten Region gemessenen Wellenlängen sind jedoch wegen des letzteren Umstandes bis auf Weiteres immer noch brauchbar, wenn man sie nur vor ihrer Bentitzung mit Hilfe einer - auf Grund der in der Tabelle I ibersichtlich zusammengestellten Zahlen für die Kadmium- und Kupferlinien (von Hartley-A deney, LiveingDewar, L. Bell und Trowbridge-Sabine) - construirten Fehlerdifferenzencurve corrigirt.

Die Messungen von Liveing und Dewar sind aber auch in dieser Region sehr genau uud verlässlich, wie aus der Vergleichung der von ibnen und von Trowbridge und Sabine gemessenen Wellenlängen der Kupferlinien deutlich hervorgeht. (Siehe Tafel I.) Die Fehlerhaftigkeit der Wellenlängen von Hartley und Adeney für $\lambda \leqq 2370$ hat glucklicherweise keinen wesentlichen Einfluss a uf meine bisherigen vergleichenden Untersuchungen und gar keinen auf deren Endergebnisse, da ich fast durchwegs die sehr verlässlichen Messungen von Liveing und Dewar und die mit ihnen für $\lambda>2370$ wirklich vergleichbaren von Hartley und Adeney benützt habe.

Der ganze diesbezügliche Einfluss besteht darin, dass in meiner "Spectralanalyse des Kadmiums" (diese Berichte 1888) 8 unter 49 Wellenlängen der VI. Gruppe, sowie 8 unter 72 Wellenlängen der V. Gruppe, also zusammen 16 unter 121 Wellenlängen durch deren, auf die oben angegebene Art bestimmten verbesserten Werthe zu ersetzen, und in die Gruppen, zu denen sie gehören, einzureilhen sind, wobei 4 von ihnen (darunter L. Bell's $2288 \cdot 01$ und $2264 \cdot 42$ ) in die V. Gruppe, die übrigen 12 dagegen (darunterL. Bell's $2329 \cdot 22,2321 \cdot 14,2264 \cdot 88,2193 \cdot 98$ und $2143 \cdot 75$ in die VI. Gruppe der Kadminunstrahlen fallen.

Ich hoffe, dass es mir, falls ich von hiezu berufener Seite die nöthige materielle Unterstiitzung finde, möglich sein wird, mit geeigneten Hilfskräften nicht nur die Fehlerdifferenzeurven, beziehungsweise Tafeln für den Übergang von den Live ing-Dewar'schen, sowie von den Hartley-Adeney'schen Zablen in 
verschiedenen Spectren zu deren möglichst verbesserten Werthen nach Rowland's Seala zu coustruiren, und meine bisherigen Vergleichungen (insolange als nicht noch genauere Messungen vorhanden sind) mit den letzteren zli wiederbolen, sondern auch rascher in der Verfolgung und Aufdeckung neuer, höchst wichtiger Beziehungen, denen ich auf der Spur bin, fortzuschreiten. Denn nicht bloss die maximale, in einem Elemente der Mendelejeff'schen Tafel vorkommende Condensationsform des primären Elementes " $b$ " steht, wie ich bereits gefunden habe, in einer einfachen Beziehung zu seiner Stellung in der Mendelejeff'schen Tafel (siehe unten!), sondern es gilt auch $\ddot{\text { bnnliches }}$ fuir die minimale in einem Elemente vorkommende Condensationsform von $" b^{4}$, sowie für die minimalen und maximalen darin auftretenden Condensationsformen des primären Elementes "a", so dass es möglich sein wird, bloss durch die Untersuchnng des Spectrums eines noch unbekannten secundären Elementes nicht nur die Reihe, sonderu auch die Gruppe der Mendelejeff'schen Tafel, welcher es angehört, und damit das approximativë Atomgewicht und die sonstigen allgemeinen Eigenschaften desselben zu bestimmen.

III.

Ich habe bereits in der Einleitung zu meiner "Spectralanalyse des Kadmiums" (diese Berichte 1888, S. 2-5) einen wichtigen, von mir im Wege der Induction gefundenen Zusammenhang. zwischen den Spectren der Elemente und ihrer Stellung in der Mendelejeff'schen Tafel mitgetheilt, von dessen Richtigkeit man sich nach den dortigen Angaben durch wirkliche Ausführung der erforderlichen Vergleichungen der Spectren mit dem Wasserspectrum von Liveing und Dewar überzeugen kann.

Die vergleichenden Zahlentabellen selbst, aus welchen dieses Inductionsgesetz hervorgeht, können, da mir zu meinen Arbeiten keine Hilfskräfte zur Verfügung stehen, erst später nach und nach pnblicirt werden. Inzwischen boffe ich, durch die direct experimentell controlirbaren Ergebnisse der vorliegenden, sowie der in Aussicht genommenen Publication über meine "Vergleichende Spectralanalyse des Kobalts und Nickels" die grosse Wichtigkeit des Gesetzes darthun za können. 
Es hat sich nämlich durch Vergleichung der Wellenlängen einer grossen Anzahl von Elementen mit den Wellenlängen des Wasserspectrums herausgestellt, dass das Spectrum eines Elementes der $n^{\text {ten }}$ Reihe der Mendelejeff'schen Tafel für $n>2$ eine Gruppe meist sehr brechbarer Strahlen enthält, deren Wellenlängen $\lambda$ dureh Multiplication mit dem Reductionsfactor $\frac{n}{n-1}$ in entsprechenden Wellenlängen $\frac{n}{n-1} \lambda=\lambda^{\prime}$ des Wasserspectrums übergehen. Die letzteren gehören dem primären Elemente " $b$ “ des Hydrogens $\mathrm{H}=\boldsymbol{b} \boldsymbol{a}_{z_{4}}$ in dem condensirten Zustande an, in sich dasselbe im Hydrogen des Wasserdampfes befindet. Die sehr reichhaltige Gruppe von Wellenlängen jener Strablen, welche das primäre Element ${ } b$ " in dem erwähnten Zustande innerhalb des Wasserdampfes aussendet, und welche von mir durch das Symbol $\left(\mathrm{H}_{2} \mathrm{O}, \mathrm{H}, b\right)$ bezeichnet wird, kann als solche durch eine Reihe von Kriterien erkannt werden, die ich bei meinen vergleichenden Untersuchnngen der Spectren des Hydrogens, Oxygens und des Wasserdampfes (Astronomische Nachrichten Nr. 2797 und diese Berichte, 1887) gefunden und in der Einleitung zu meiner "Spectralanalyse des Magnesiums und der Kohle" (diese Berichte, 1887, S. 3) ibersichtlich zusammengestellt habe. Nach diesen Kriterien müssen alle Wellenlängen $\lambda^{\prime}$ dieser Gruppe $\left(\mathrm{H}_{2} \mathrm{O}, \mathrm{H}, \boldsymbol{b}\right)$, wenn sie mit $\frac{5}{4}$ multiplicirt werden, in virtuelle Wellenlängen $\frac{5}{4} \lambda^{\prime}=\lambda^{\prime \prime}$ des Hydrogens tibergehen, welchen dem primären Stoffe $" b$ " in jener chemischen Condensationsform angehören, in welcher er auch im freien Hydrogen vorkommt. Die zu diesen Wellenlängen gehörigen Strahlen hängen hinsichtlich ihrer Sichtbarkeit von der jeweiligen physikalischen Modification der betreffenden Condensationsform ab. Die Gruppe, zu welcher die Strahlen $\lambda^{\prime \prime}$ gehören, wird von mir durch das Symbol $(\mathrm{H}, b)$ dargestellt. Alle Wellenlängen $\lambda^{\prime \prime}$ derselben lassen sich durch Multiplication mit $\frac{46}{41}$ und $\frac{70}{59}$ in virtuelle Wellenlängen des Oxygens verwandeln, welche, beziehungsweise mit $\frac{23}{32}$ and $\frac{21}{32}$ multiplicirt, in homologe Wellenlängen $\frac{23}{32} \cdot \frac{46}{41} \lambda^{\prime \prime}$ und $\frac{21}{32} \cdot \frac{70}{59} \lambda^{\prime \prime}$ des Wasser- 
dampfes, übergehen. Die Wellenlängen $\frac{46}{41} \lambda^{\prime \prime}$ und $\frac{70}{59} \lambda^{\prime \prime}$ gehören ganz bestimmten Condensationsformon des primären Elementes "b" an, welche in individuellen physikalischen Modificationen auch im Oxygen vorkommen. Das Oxygen ist nämlich nach meinen vergleichenden Untersuchungen ein zusammengesetzter Körper, welcher zunächst aus einem für dieses secundäre Element besonders charakteristischen Stoffe $O^{\prime}$ und aus dem Hydrogen in der modificirten Form $\mathrm{H}^{\prime}$ besteht, in der es das sogenannte zusammengesetzte Linienspectrum erzengt: $\mathrm{O}=\mathrm{H}^{\prime} . \mathrm{O}^{\prime}$. (Sollten die namentlich in den 0-Spectren älterer Beohachter, wie Plü ck er ete., vorkommenden $\mathrm{H}^{\prime}$-Strahlen nur von sehr verdïnntem, dem Oxygen bloss beigemischtem Hydrogen herriihren, so wiirde 0 einfach mit $O^{\prime}$ identisch sein). Der Stoff ${ }^{\prime \prime 6}$ besteht ans vier Volumen des primären Elementes $„ b$ " und fünf Volnmen eines Stoffes $\mathrm{O}^{\prime \prime}$, welcher seinerseits merkwürdiger Weise ganz ähnlich zusammengesetzt ist, indem er wieder vier Volumtheile von " $b$ " und fünf Volumtheile des primären Elementes "c" enthält.

Das letztere ist, wie ich erst unlängst gefunden habe, nur eine bestimmte chemische Condensationsform des primären Elementes " $a^{\prime}$ des Hydrogens $\mathrm{H}=\boldsymbol{b} a_{4}$.

a) Die Wellenlängen $\frac{46}{41} \lambda^{\prime \prime}$, welche durch Multiplication mit $\frac{46}{41}$ aus den Wellenlängen $\lambda^{\prime \prime}$ der Gruppe $(H, b)$ gewonnen werden, gehören dem primären Elemente " $b$ " in dem Zustande an, in welchem es, mit dem Stoffe $0^{\prime \prime}$ verbunden, in dem Stoffe $O^{\prime}=b_{4} O^{\prime \prime}$ innerhalb des Oxygens enthalten ist. Diese Gruppe von Wellenlängen wird von mir mit dem Symbole $\left(0,0^{\prime}, b\right)$ bezeichnet. Der in diesem Zustande befindliche Stoff wird, wenn sich das Oxygen mit dem Hydrogen zu Wasserdampf verbindet, in bestimmtem Versältnisse condensirt, und sendet dann Strahlen innerhalb des Wasserdampfes ans, deren Wellenlängen durch Multiplication mit 23

$\frac{23}{32}$ aus den entsprechenden Wellenlängen der Gruppe $\left(0, O^{\prime}, b\right)$ abgeleitet werden können. Die Wellenlängen dieser Gruppe des Wasserspectrums werde i ron mir durch das $\mathrm{Symbol}\left(\mathrm{H}_{2} \mathrm{O}, \mathrm{O}, \mathrm{O}^{\prime}, b\right)$ angedentet. 
b) Die Wellenlängen $\frac{70}{59} \lambda^{\prime \prime}$, welche durch Maltiplication mit $\frac{70}{59}$ aus den Wellenlängen $\lambda^{\prime \prime}$ der Gruppe $(H, b)$ erhalten werden, gehören dem primären Elemente,$b$ " in dem von dem vorigen ganz verschiedenen chemischen Zustande an, in welchem es mit "c $c^{\prime \prime}$ zum Körper $0^{\prime \prime}=b_{4} c_{5}$ vereinigt, innerhalb des Körpers $\mathrm{O}^{\prime}=b_{4} \mathrm{O}^{\prime \prime}{ }_{5}$ im Oxygen vorkommt. Die Gruppe dieser Wellenlängen wird von mir durch das Symbol $\left(0,0^{\prime}, 0^{\prime \prime}, b\right)$ dargestellt.

Werden die Wellenlängen $\frac{70}{59} \lambda^{\prime \prime}$ dieser Gruppe $\left(0,0^{\prime}, 0^{\prime \prime}, b\right)$ mit $\frac{21}{32}$ multiplicirt, so werden sie dadurch in rhythmisch entsprechende Wellenlängen $\frac{21}{32} \cdot \frac{70}{59} \lambda^{\prime \prime}$ des Wasserspectrums transformirt, und bilden eine von mir mit $\left(\mathrm{H}_{2} \mathrm{O}, 0, \mathrm{O}^{\prime}, \mathrm{O}^{\prime \prime}, b\right)$ bezeichnete Gruppe, welche dem Elemente " $b$ " des Stoffes $O^{\prime \prime}=b_{4} c_{5}$ in dem Zustande angehört, in welchem es sich im Stoffe $0^{\prime}=b_{4} O^{\prime \prime}{ }_{5}$ des Oxygens innerhalb des Wasserdampfes befindet. Zum besseren Verständnisse der sogenannten virtuellen Strahlen, welche zum Hydrogen und Oxygen, oder vielmehr zu verschiedenen physikalischen Modificationen solcher Condensationsformen der primären Elemente $a$ und $b$ des Hydrogens $\mathrm{H}=b a_{4}$ gehören, die in individuellen physikalischen Modificationen auch im $\mathrm{H}$ und $O$ vorkommen, verweise ich auf die Einleitung zu meiner "Spectralanalyse des Kadmiums". (Diese Berichte, 1888, S. 6-8.)

Kehren wir nach dieser kleinen, zum leichteren Verständnisse des Folgenden dienenden Erläuterung wieder zu unserem Hauptgegenstande zurïck.

Das in Rede stehende Inductionsgesetz, betreffend den Zusammenhang zwischen den Spectren der Elemente und ihrer Stellung in der Mendelejeff'schen Tafel, kann in eine Form gebracht werden, in welcher es direct experimentell geprüft, und eventuell eine werthvolle Directive für Experimentaluntersuchungen werden kann, welche auf Entdeckung noch -unbekannter secundärer Elemente abzielen, deren Existenz, approximatives Atomgewicht und allgemeine Eigensehaften zwar durch das periodiscbe Gesetz angezeigt werden, deren Plätze in der 
Mendelejeff'schen Tafel jedoch zur Zeit noch leer stehen und ihrer künftigen Inhaber harren.

Angenommen, ein Element $X$ der $n$ ten Reihe der Mendelejeff'schen Tafel komme in einem Stoffe $A$ ohne chemische Volumänderung, d. b. ohne chemische Condensation oder Dilatation vor; es sei z. B. "A" ein bekanntes, aber nicht ganz reines Element und " $X^{\prime}$ eine Spur von einem noch unbekannten Elemente, durch welches das erstere verunreinigt wird. Dann muss das Spectrum des Stoffes $A$, insbesondere der ultraviolette Theil desselben, nach dem obigen Inductionsgesetze eine Gruppe von Strahlen enthalten, deren Wellenlängen $\lambda$ mit $\frac{n}{n-1}$ multiplicirt, in Wellenlängen des Wasserspectrums übergehen, welche eine Partialgruppe der sehr ausgedehnten Gruppe $\left(\mathrm{H}_{2} \mathrm{O}, \mathrm{H}, b\right)$ bilden, und als solche mittelst der Kriterien $\frac{5}{4}\left(\mathrm{H}_{2} \mathrm{O}, \mathrm{H}, \boldsymbol{b}\right)=(\mathrm{H}, b)$

$$
\begin{aligned}
& \frac{23}{32} \cdot \frac{46}{41}(\mathrm{H}, b)=\left(\mathrm{H}_{2} \mathrm{O}, 0, \mathrm{O}^{\prime}, b\right) \text { Wasserspectrum, } \\
& \frac{21}{32} \cdot \frac{70}{59}(\mathrm{H}, b)=\left(\mathrm{H}_{2} \mathrm{O}, \mathrm{O}, \mathrm{O}^{\prime}, \mathrm{O}^{\prime \prime}, b\right) \text { Wasserspectrum }
\end{aligned}
$$

erkannt werden können.

Wiirde das Element $X$ im Stoffe $\boldsymbol{A}$ nicht, wie oben vorausgesetzt wurde, ohne Condensation, sondern in einer chemischen Verbindung mit einem andern Elemente $Y$ vorkommen, welche nur unter Condensation der betheiligten Elemente im Verhältnisse $m: p\left(\frac{m}{p}=\frac{1}{2}, \frac{2}{3}, \frac{2}{5}, \frac{2}{7} \ldots\right)$ zu Stande kommen kann, so würden sämmtliche Wellenlängen $\lambda$ desselben [also auch die der charakteristischen Gruppe $\frac{n}{n-1} \lambda=$ $=\left(\mathrm{H}_{2} \mathrm{O}, \mathrm{H}, b\right)$ Wasserspectrum] nach meinem Fundamentalgesetze über die Veränderung der Wellenlängen von Componenten bei Bildung einer chemischen Verbindung, rational im Verhältnisse $m: p$ geändert, und in Wellenlängen von der Form $\frac{m}{p} \lambda=l$ übergeftuhrt werden, abgesehen von anderen gesetz- 
mässigen rationalen Veränderungen untergeordneter Gruppen von Wellenlängen.

Die charakteristische Gruppe des freien oder wenigstens ohne Condensation gebundenen Elementes $X: \frac{n}{n-1} \lambda=\left(\mathrm{H}_{2} \mathrm{O}, \mathrm{H}, b\right)$ wtirde sich in eine Gruppe $\frac{n}{n-1} \cdot \frac{p}{m} l=\left(\mathrm{H}_{2} \mathrm{O}, \mathrm{H}, b\right)$ von Wellenlängen $l$ verwandeln, welche mit $\frac{n}{n-1} \cdot \frac{p}{m}$ (und nicht mit $\left.\frac{n}{n-1}\right)$ multiplicirt, in Wellenlängen der Gruppe $\left(\mathrm{H}_{2} \mathrm{O}, \mathrm{H}, b\right)$ übergehen wiirden.

Der hier auftretende Factor $\frac{n}{n-1}, \frac{p}{m}$ könnte nicht die Form $\frac{n^{\prime}}{n^{\prime}-1}\left(n^{\prime}=3,4,5 \ldots 12 \ldots, n^{\prime} \lesseqgtr n\right)$ annehmen; denn wäre $\frac{n}{n-1} \cdot \frac{p}{m}=\frac{n^{\prime}}{n^{\prime}-1}$, so müsste $\frac{n}{n-1}: \frac{n^{\prime}}{n^{\prime}-1}=\frac{m}{p}$ einer der einfachen chemischen Condensationsfactoren $\frac{1}{2}, \frac{2}{3}, \frac{2}{5}, \frac{2}{7} \ldots$. sein, was nicht der Fall ist, wie man sich leicht durch wirkliche Division zweier verschiedener Zahlen der Reihe $\frac{3}{2}, \frac{4}{3}, \frac{5}{4}$, $\frac{5}{5}, \frac{7}{6}, \frac{8}{7}, \frac{9}{8}, \frac{10}{9}, \frac{11}{10}, \frac{12}{11}$ überzeugen kann.

Daraus folgt, dass man das Inductionsgesetz auch umkehren, und demselben die nachstehende, für die Anwendung sehr zweckmässige Fassung geben kann:

"Wenn ein Stoff $A$ ein Linienspectrum liefert, in welchem besonders im Ultraviolett mehr oder weniger zahlreiche Strahlen vorkommen, deren Wellenlängen $\lambda$ durch Multiplication mit einem Bruche von der Form $\frac{n}{n-1}\left(n>2, \frac{n}{n-1}=\frac{3}{2}, \frac{4}{3}, \frac{5}{4}\right.$, $\left.\frac{6}{5}, \frac{7}{6}, \frac{8}{7}, \frac{9}{8}, \frac{10}{9} \cdot \frac{11}{10}, \frac{12}{11}\right)^{n-1}$ in rhythmisch entsprechende Wellenlängen $\lambda^{\prime}=\frac{n}{n-1} \lambda$ des Wasserspectrums verwandelt werden können, die sich mittelst der bekannten Kryterien als zur Gruppe $\left(\mathrm{H}_{2} \mathrm{O}, \mathrm{H}, b\right)$ gehörig nachweisen lassen, so enthält der Stoff $\boldsymbol{A}$ zum Mindesten eine Spur eines bekannten oder noch unbekannten Elementes $\boldsymbol{X}$ der $\boldsymbol{n}^{\text {ten }}$ Reihe der Mendelejeff'schen Tafel ohne chemische Condensation". 
IV.

Verglejeht man die von Hartley und Adeney gemessenen Wellenlängen der Spectren des Tellurs, des Antimons und des Kupfers im Ultraviolett ohne Rücksicht auf die äussere Erscheinung der zugehörigen Linien (siehe Nr. I!), so findet man die in derTabelle II zusammengestellten Übereinstimmungen. Die ersten zwei Colonnen der Tafel geben die betreffenden Wellenlängen des Tellurs und Antimons, die dritte die eventuell ubereinstimmenden des Kupfers, die vierte die Mittelwerthe der in einer und derselben Zeile stehenden entsprechenden Zahlen, die fünfte endlich die adoptirten Werthe $\lambda$ der Wellenlängen jener Strablen, welche zugleich im Spectrum des Tellurs und Antimons, eventuell anch im Spectrum des Kupfers vorkommen. Für $\lambda>2370$ (Angst. Sc.) stimmen die adoptirten Wellenlängen mit den Mittelwerthen der von Hartley und Adeney gegebenen Zahlen überein; für $\lambda \leqq 2370$ dagegen sind statt der Mittelwerthe der vierten Colonne deren auf die oben in $\mathrm{Nr}$. II angegebene Weise corrigirte Werthe als die der Wahrheit am nächsten kommenden Wellenlängen adoptirt worden.

Die obigen Übereinstimmungen sind so genau, die Zwischenräume zwischen den Linien, welche in den Spectren des Tellurs, Antimons und Kupfers den übereinstimmenden zun ächst liegen, relativ so gross, dass eine blosse Zufälligkeit der Coincidenzen sehr unwahrscheinlich ist. Überdies dürften das Tellur und andere damit nahe verwandte Metalle häufig Begleiter des atomanalogen Schwefels in den schwefelhältigen Antimon- und Kupfererzen (Grauspiessglanzerz $\mathrm{Sb}_{2} \mathrm{~S}_{3}$, Kupferglanz $\mathrm{Ca}_{2} \mathrm{~S}$, Kupferkies oder Buntkupfererz $\mathrm{CuFeS}_{2}$ etc.) sein; das Vorkommen von Spuren des Tellurs und ihm ähnlicher, vielleicht noch unbekannter Metalle in dem aus solchen Erzen gewonnenen Antimon und Kupfer, sowie das Auftreten gemeinsamer Strahlen in den Spectren der letzteren Elemente und in jenem des Tellurs wären nothwendige Folgen davon, somit leicht erklärlich. Endlich ist noch zu beachten, dass das Atomgewicht des Tellurs nach seiner Stellung in der siebenten Reihe der Mendelejeff'schen Tafel (Gruppe VI!) ungefähr 125 betragen sollte, während es mittelst verschiedener Methoden verschieden, u. zw. meist viel grösser, zu 128 und mehr, gefunden wird. 
Berzelius fand es 1812 und $1818 \mathrm{zu} 128 \cdot 9,1832 \mathrm{zu} 128 \cdot 3$, indem er Tellur mittelst Salpetersäıre oxydirte und das beim Gliihen zurückgebliebene Telluriumdioxyd wog; v. Ha u er erhielt dafiir 1857 mittelst der Analyse des Kaliumtelluriumbromides 127.9. Dr. B. Brauner endlich erhielt es aus der Synthese des Telluriumdioxydes und des Telluriumsulfates zu 125-126, dagegen aus der Analyse des Telluriumtetrabromides und Telluriumdibromides zu 128 und mehr $(0=16)$.

Dieses eigenartige Verhalten des Tellurs bei den Versuchen, sein Atomgewicht mittelst verschiedener Methoden za bestimmen, gibt viel zu denken, und spricht namentlich dafür, dass das bisherige "Tellur" noch kein einheitlicher Körper ist, sondern aus einem noch unbekannten ganz reinen Tellur und kleinen Beimengungen besteht, welche dem reinen Tellur nahe verwandt und desshalb von ihm sehr schwer zu trennen sind (siehe Dr. B. Brauner: "Experimental researches on the periodic law", Part I, Tellurium, im Journal of the Chemical Society, Jnly 1889).

Die erwähnten Beimengungen dürften zugleich mit dem reinen Tellur als hartnäckige Begleiter des Schwefels in den Antimon- und Kupfererzen vorkommen und aus diesen in das gewonnene Antimon und Kupfer ibergehen.

Erwägungen dieser Art, welche es wahrscheinlich machen, dass die gemeinsamen Strahlen des Tellurs, Antimons und Kupfers im Ultraviolett theils dem noch unbekannten rein en Tellur, th eils den ebensowenig oder vielmehr noch weniger bekannten Begleitern desselben gehören, haben mich bestimmt, die genannten Strahlen eingehend zu prüfen.

Indem ich nun die Wellenlängen derselben auf Grund des in Nr. III besprochenen, von mir entdeckten Inductionsgesetzes zunächst daraufhin untersuchte, ob und welche derselben sich durch Multiplication mit rationalen Zahlen von der Form $\frac{n}{n-1}$ $(n=3,4,5,6.7,8,9,10,11,12)$ in Wellenlängen der Gruppe $\left(\mathrm{H}_{2} \mathrm{O}, \mathrm{H}, b\right)$ des Wasserspectrums transformiren lassen, fand ich unter Anderem, dass die nachstehenden Wellenlängen $\lambda$ unter ihnen eine charakteristische Gruppe bilden, deren Zahlen $\lambda$ durch Multiplication mit $\frac{11}{10}$ in rhythmisch entsprechende Welienlängen 
$\lambda^{\prime}=\frac{11}{10} \lambda$ des Wasserspectrums übergehen, welche sich durch die bekannten Kriterien als zur Gruppe $\left(\mathrm{H}_{2} \mathrm{O}, \mathrm{H}, b\right)$ gehörig crweisen.

\section{Charakteristische Gruppe I}

ibereinstimmender Strahlen des Tellurs und Antimons, eventuell auch des Kupfers.

Adoptirte Wellenlängen. Angstr. Scala ${ }^{t}$ :

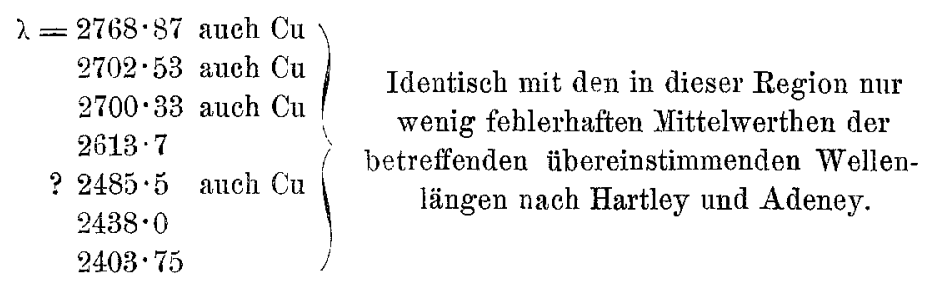

Adoptirte Wellenlängen, erhalten rlurch Correctur der rechts stehenden entsprechenden Mittelwerthe übereinstimmender Wellenlängen nach Hartley und Adeney (siehe Nr. II und Tafel I):

$$
\begin{aligned}
& \lambda= 2369 \cdot 5 \text { anch } \mathrm{Cu} \\
& 2279 \cdot 32 \\
& 2241 \cdot 76 \text { auch } \mathrm{Cu} ? \\
& 2229 \cdot 73 \text { auch } \mathrm{Cu} \\
& 2227 \cdot 43 \text { auch } \mathrm{Cu} \\
& 2221 \cdot 83 \\
& 2214 \cdot 93 \text { auch } \mathrm{Cu} \\
& 221403 \text { anch } \mathrm{Cu} \\
& \\
& 2159 \cdot 71
\end{aligned}
$$

\section{Mittelwerthe übereinstimmender Wellenlängen von Hartley und Adeney, welche in dieser Region den links stehenden richtigeren Werthen entsprechen:}

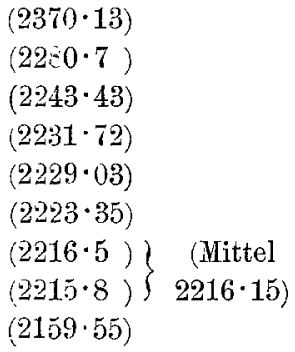

Die Genauigkeit, mit welcher die obigen Wellenlängen den sie kennzeichnenden rhythmischen Beziehungen zum Wasserspectrum genügen, ist aus den ohne weitere Erläuterung verständlichen Tabellen III, III $a$ ) und III $b$ ) ersichtlich, wenn man nur beachtet, dass die Tabellen III $a$ ) und III $b$ ) eigentlich blosse Abzweigungen und Fortsetzungen der Tabelle III bilden, indem

1 Die obigen, auf Angström's Scala bezüglichen Wellenlängen $\lambda$ können leicht auf die Rowland'sche Scala reducirt werden, indem man zu jeder derselben $1 / 6000$ ihres Betrages hinzufiigt. 
ihre ersten Colounen mit der vierten Colonne der letzteren identisch sind.

Aus dem Vorhergehenden folgt sofort auf Grund des in Nr. III ausgesprochenen Satzes über den Zusammenhang zwischen den Spectren der Elemente und ihrer Stellung in der Mendelejeff'schen Tafel, dass

"die Strahlen der charakteristischen Gruppe (I) einem Elemente $X$ der 11. Reihe der Mendelejeff'schen Tafel angehören, welches im bisherigen T $\in l l u r$ und Antimon und zagleich auch im Kapfer ohne chemische Condensation enthalten ist".

Die Elemente der 11. Reihe sind aber, wenn wir überhaupt ein Element der $\boldsymbol{m}_{\text {ten }}$ Gruppe und $\boldsymbol{n}$ ten Reihe mit $\boldsymbol{R}^{m, n}$ bezeichnen, die folgenden : $\boldsymbol{R}^{1,11}=\mathrm{Au}$ Gold, $\boldsymbol{R}^{2,11}=\mathrm{Hg}$ Quecksilber, $R^{3,11}=$ $=\mathrm{Tl}$ Thallium, $\boldsymbol{R}^{4,11}=\mathrm{Pb}$ Blei, $\boldsymbol{R}^{5,11}=\mathrm{Bi}$ Wismuth, $\boldsymbol{R}^{6,11}=212$ ungefähr (unbekannt, Dr.B. Brau ner's Austriacum?), $R^{7,11}=215$ ungefähr (unbekannt) und $\boldsymbol{R}^{8,11}=119$ ungefähr (unbekannt, Dahll's Norwegium?).

Das gefundene, vorläufig nur spectralanalytisch durch die zugehörige charakteristische Strahlengruppe (I) definirte Element $X$ der 11. Reihe kann nun, wie leicht nachgewiesen werden kann, keines der bereits bekannten Elemente dieser Reihe sein. Denn. wenn $X$ mit einem der bekannten Elemente derselben identisch wäre, so müissten sämmtliche Linien der charakteristischen Gruppe im ultravioletten Spectrum des Ietzteren deutlich si ch tb a r sein, nachdem schon eine blosse Spur von $X$ im Tellur und Antimon die betreffenden Linien auf der photographischen Platte erzeugt.

Dem entgegen enthält jedoch das Gold-Spectrum im Ultraviolett uiberhanpt keinen der übereinstimmenden Strahlen des Tellurs, Antimons und Kupfers, welche wir in der Tafel II übersichtlich zusammengestellt haben; das Quecksilber-Spectrum hat nach Hartley und Adeney nur einen einzigen Strahl $\lambda 2231 \cdot 0$, dessen nach den Angaben in $\mathrm{Nr}$. II mit Hilfe der Tafe I I rectificirte Wellenlänge $2229 \cdot 46$ der adoptirten Wellenlänge $2229 \cdot 73$ der charakterischen Gruppe sehr nahe liegt; das Thallium-Spectrum hat nur zwei Strahlen: $2700 \cdot 1,2243 \cdot 7$ rectificirt: $2242 \cdot 03$, nach $\mathrm{Hartley}$ etc., welche den adoptirten Strahlen $2700 \cdot 33$ und $2241 \cdot 76$ der charakteristischen Gruppe sehr nahe kommen; das Bleispectrum bat nur einen derartigen 
Strahl: $2613 \cdot 7$ nach Liveing und Dewar, $2613 \cdot 4$ nach Hartley; und das Wismuth-Spectrum wieder nur zwei: 2231.4, $2229 \cdot 1$ nach Hartley, rectificirt: $2229 \cdot 86$ und $2227 \cdot 5$, welche mit adoptirten Strahlen der charakteristischen Gruppe 2229.73 und $2227 \cdot 43$ nahezu uibereinstimmen.

"Das Element $X$ kann daher nur eines der noch unbekannten Elemente der elften Reihe: $R^{6,11}=212$ ungefähr (Dr. B. Brauner's Austriacum?), $R^{7,11}=215$ ungefähr, $R^{8,11}=219$ ungefähr (D ahll's Norwegium?) sein".

Berïcksichtigen wir nun noch, dass das Element $X$, welches nach dem Obigen der VI., VII. oder VIII. Gruppe der Mendelejeff'schen Tafel angehört, jedenfalls dem Antimon $=\boldsymbol{R}^{5,7}$ (in der V. Gruppe), besonders aber dem Tellur $=\boldsymbol{R}^{6,7}$ (in der VI. Gruppe) nahe verwandt sein muss, weil es sonst nicht so hartnäckig die meisten für das Antimon und Tellur als charakteristisch angesehenen Reactionen mit den letzteren mitmachen könnte, so erkennen wir, dass $X$ höchstwahrscheinlich derselb en VI. Gruppe wie das Tellur angehören, also mit dem Elemente $\boldsymbol{R}^{6,11}=212$ ungefähr, Dr. B. Brauner's Austriacum?, identisch sein dürfte.

Im Kupfer spielt $X$ jedenfalls die Rolle eines elektro-negativen Elementes. Das Kupfer ist höchst wahrscheinlich eine Verbindung oder vielmehr eine Legirung eines stark alkalisch reagirenden noch unbekannten Elementes $R^{1,5}$, welchem eigentlich der jetzt noch dem Kupfer prorisorisch zugewiesene Platz in der I. Gruppe der Mendelejeff'schen Tafel gebührt, mit anderen elektronegativen metallischen Elementen, unter welchen sich nach dem Obigen jedenfalls das Element $X$ (Brauner's Austriacum?) befindet. Da das unbekannte alkalische Element $R^{1,5}$ des Kupfers zufolge seiner Stellung zwischen Kalium und Rubidium und seiner besonders nahen Verwandtschaft mit dem Natrium sehr leicht, und zwar leichter als das Kalium schmelzbar sein muss, das uns bekannte Kupfer jedoch erst bei cirea $1000^{\circ}$ schmilzt, so muss (indem hier von dem im Kupfer wohl nur in sehr geringer Menge vorkommenden Elemente $X$ abgesehen werden kann) der elektronegative Hauptbestandtheil des Kupfers ${ }^{1}$ ein schwer schmelzbares Element sein. Für das 1 mindestens. 
Vorkommen eines solchen elektronegativen Bestandtheiles im Kupfer neben dem eigentlichen, stark alkalischen Hauptbestandtheil $R^{1,5}$ (= $=55$ ungefähr?; wegen der Beziehung: $\boldsymbol{R}^{1,5}$ zu $\boldsymbol{K}$, wie $\mathrm{Na} \mathrm{zu} \mathrm{Li)} \mathrm{spricht} \mathrm{auch} \mathrm{deutlich} \mathrm{die} \mathrm{Existenz} \mathrm{des} \mathrm{Kupfer-}$ wasserstoffes, welcher nur desshalb zu Stande kommen kann, weil der elektronegative Bestandtheil des Kupfers die sonst sehr g ering e Affinität des stark alkalischen Elementes $R^{1,5}$ gegen Wasserstoff durch Induction soweit erhöht, dass auch dieser a $1 \mathrm{k}$ a l is che Bestandtheil den Wasserstoff, allerdings nur höchstens bis zu einer Temperatur von circa $60^{\circ} \mathrm{C}$. festzuhalten vermag. ${ }^{1}$

Die bis jetzt näher betrachteten Strahlen der charakteristischen Gruppe (I) bilden nur einen Theil der Strahlen des Elementes $X$, von welchen sich noch manche andere unter den gemeinsamen Strahlen des Tellurs und Antimons befinden dürften.

Insbesondere dürften die Strahlen: $\lambda=2611 \cdot 3,2549 \cdot 75$, $2479 \cdot 5$ und $2462 \cdot 0$ auch noch Strahlen des Elementes $X$ sein. Dieselben bilden mit dem Strahle $\lambda=2613 \cdot 7$ eine bemerkenswerthe Gruppe, welehe wir die Gruppe If nennen wollen.

Ihre Wellenlängen $\lambda$ besitzen alle Merkmale von Strahlen, welche einer ganz bestimmten Condensationsform des primären Elementes " $\alpha$ " angehören. Sie gehen nämlich mit $\frac{3}{2}$ multiplicirt in Wellenlängen $\frac{3}{2} \lambda=\lambda_{1}$ der Gruppe $(\mathrm{H}, a)$ über, welche dem

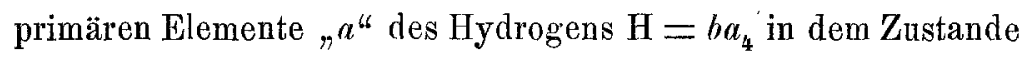
gehören, in welchem es sich im freien Hydrogen befindet. Die letzteren Wellenlängen $\lambda_{1}$ genügen in der That den diesbezilg. lichen Kriterien; indem $\frac{19}{30} \lambda_{1}, \frac{3}{4} \lambda_{1}$ und $\frac{56}{75} \lambda_{1}$ rhythmisch entsprechende Wellenlängen des Wasserspectrums und überdies $\frac{2}{3} \lambda_{1}=\lambda$ Wellenlängen sind, welche mit homologen Zahlen des Wasserspectrums iibereinstimmen. (S. meine "Mathem. Spectralanalyse des Magnesiums und der Kohle", diese Berichte 1887, S. 2,3 und 5 !)

1 Das alkalische Element $R^{1,5}$ würde füir sich allein blos $R_{2}^{1,5} H$ bilden können, ähnlich wie das $N a$ blos $N a_{2} H$ bildet, während $C u: C u_{2} H_{2}$ liefert. 
Die oben angefubrten Beziehungen der Wellenlängen $\lambda$ zum Wasserspectrum sind ans den Tabellen IV und IV $a$ ersichtlich, von welchen die letztere nur eine Fortsetzung der ersteren bildet, indem die 1. Colonne der Tabelle IV $a$ mit der 3. Colonne der Tabelle IV identisch ist.

Da nun der Strahl $\lambda=2613 \cdot 7$ sowohl dieser, als auch der charakteristischen Gruppe (I) angehört, mithin ein empirischer Knotenstrahl (Verzweigungsstrahl) der beiden Gruppen ist, so wird er, im Falle er auch ein wirklicher Knotenstrahl ist, von einem Atomtheilchen erzeugt, welches die den beiden Gruppen cntsprechenden Condensationsformen der primären Elemente „ $a^{\text {c }}$ nnd,$b$ " materiell mit einander verbindet; dies ist aber nur möglich, wenn beide Condensationsformen als integrirende Bestandtheile an der Structur eines und desselben secundären Elementes, also hier des Elementes $X$ betheiligt sind. Ist dies aber der Fall, so gehören die beiden entsprechenden Strahlengruppen I und II diesem Elemente an, wie oben behauptet wurde.

Wenn wir schliesslich alles bisher Gefundene zusammenfassen, so können wir als Endergebniss unserer Analyse den nachstehenden

\section{B e f u n d}

aussprechen: „Die bisherigen Elemente Tellur, Antimon und Kupfer enthalten Spuren eines neuen, noch unbekannten Elementes $X$ der 11. Reihe der Mendelejeff'schen Tafel. Das letztere ist einerseits dem Tellur, anderseits dem Wismuth nahe verwandt und höchst wahrscheinlich identisch mit dem Elemente der VI. Gruppe und 11. Reihe: $\boldsymbol{R}^{6,11}$ von dem ungefähren Atomgewichte 212 , also mit dem von Dr. B. Brauner neuestens im Tellur entdeckten "Austriacum".

Das neue Element besitzt ein Spectrum, welches im Ultraviolett unter anderen die Strahlen:

$\begin{aligned} \lambda=2768 \cdot 9 \text { Angstr. Scall } & =2769 \cdot 3 \text { in Rowland's Scala } \\ 2702 \cdot 5 & =2703 \cdot 0 \\ 2700 \cdot 3 & =2700 \cdot 8 \\ 2613 \cdot 7 & =2614 \cdot 1 \\ ? 2485 \cdot 5 & =? 2485 \cdot 9 \\ 2438 \cdot 0 & =2438 \cdot 4\end{aligned}$




$\begin{aligned} \lambda=2403 \cdot 8 \text { Angstr. Scala } & =2404 \cdot 2 \text { in Rowland's Sca'it } \\ 2369 \cdot 5 & =2369 \cdot 9 \\ 2279 \cdot 3 & =2279 \cdot 7 \\ 22241 \cdot 8 & =22242 \cdot 1 \\ 2229 \cdot 7 & =2230 \cdot 1 \\ 2227 \cdot 4 & =2227 \cdot 8 \\ 2221 \cdot 8 & =2222 \cdot 2 \\ \{2214 \cdot 9 & =2215 \cdot 3 \\ 2214 \cdot 0 & =2214 \cdot 4 \\ 2159 \cdot 7 & =2160 \cdot 0\end{aligned}$

und wahrscheinlich auch die Strahlen:

$$
\begin{aligned}
\lambda=2611 \cdot 3 \text { Angstr. Scala } & =2611 \cdot 7 \text { in Rowland's Scala } \\
2549 \cdot 8 & =2550 \cdot 2 \\
2479 \cdot 5 & =2479 \cdot 9 \\
2462 \cdot 0 & =2462 \cdot 4
\end{aligned}
$$

enthält.

Ich habe die Hauptergebnisse meiner bisherigen vergleichenden Untersuchung des Tellur-, Antimon- und Kupferspectrums im Ultraviolett Herrn Dr. B. Brauner in Prag in einem besonderen Schreiben unterm 30. Juli 1889 mitgetheilt, um ihn mit Bezugnahme auf seine im "Journal of the Chemical Society", London und in den "Chemical News" vom 6. Juni 1889 veröffentlichten "Experimental researches on the periodic law",Part I, ,Tellurium", darauf aufmerksam zu machen, dass sein im Tellur neu entdecktes "Austriacum" nicht bloss in diesem, sondern auch im Antimon und im Kupfer vorkommt, falls mein Element $X$ der 11. Reihe der Mendelejeff'schen Tafel wirklich mit seinem Austriacum identisch sein sollte, wie es den Anschein hat; und ich schlug ihm zur Feststellung dieser Identität vor, eine kleine Probe seines Austriacums an Herrn Prof. G. D. Liveing in Cambridge, England, zu senden, damit derselbe das Spectrum des neuen Elementes, insbesondere dessen ultravioletten Theil bestimmen und so entscheiden könnte, ob die von mir vorhergesagten Strahlen des Elementes $X$ sich wirklich in diesem Spectrum vorfinden. (Die für die letzteren angegebenen Wellenlängen waren einstweilen die aus den Messungen von Hartley und Adeney 
unmittelbar fliessenden, da ich die für einen Theil derselben erforderlichen Correcturen in einem darauf folgenden Schreiben zu erläutern und mitzutheilen beabsichtigte, falls Herr Dr. Brauner auf meinen Vorschlag eingehen würde.)

Als ich kurz darauf Herrn Dr. Bra uner, der mir persönlich bis dahin ganz unbekannt war, in seinem Laboratorium aufsuchte, fand ich ihn in grosser Aufregung wegen meines Briefes, und ich war höchst überrascht, als er mir mittheilte, dass er ganz unabhängig von mir auf experimentellern Wege zu denselben Resultaten, wie ich, gelangt sei; doch sei es ihm bis jetzt noch nicht gelungen, das Austriacum aus dem Antimon und Kupfer völlig zu isoliren.

Diese merkwïrdige Ubereinstimmung von auf ganz verschiedenen Wegen erhaltener Resultaten zweier Forscher, welche bis dahin, obzwar in derselben Stadt lebend, ganz ausser aller persönlichen Berührung gebliebeu waren, ist gewiss nicht nur an sich von hohem Interesse, sondern dürfte auch geeignet sein, die Fruchtbarkeit und reale Bedeutung meiner Theorie, sowie insbesondere die Wichtigkeit des von mir im Wege der Induction gefundenen Zusammenhanges zwischen den Spectren derElemente und ihrer Stellung in der Mendelejeff'schen Tafel ad oculos zu demonstriren. 
A. Gri ti $n$ wa $1 \mathrm{~d}$,

Tabelle I. (Siehe Seite 8.)

\begin{tabular}{|c|c|c|c|c|c|c|c|}
\hline \multicolumn{2}{|c|}{ L. Bell } & \multicolumn{2}{|c|}{$\begin{array}{l}\text { Trowbridge } \\
\text { und Sabine }\end{array}$} & \multicolumn{2}{|c|}{$\begin{array}{l}\text { Liveing und } \\
\text { Dewar }\end{array}$} & \multicolumn{2}{|c|}{$\begin{array}{l}\text { Hartley und } \\
\text { Adeney }\end{array}$} \\
\hline $\begin{array}{l}\text { Rowl. } \\
\text { Scala }\end{array}$ & $\begin{array}{c}\text { Angst. } \\
\text { Scala }\end{array}$ & $\begin{array}{l}\text { Rowl. } \\
\text { Scala }\end{array}$ & $\mid \begin{array}{c}\text { Angst. } \\
\text { Scala }\end{array}$ & Angström & a's Scala & Angströr & m's Scala \\
\hline \multicolumn{2}{|c|}{$\mathrm{Kad} \mathrm{mi} \mathrm{u} \mathrm{m}$} & \multicolumn{2}{|c|}{ Kupfer } & Kadmium & Kupfer & Kadmium & Kupfer \\
\hline $\begin{array}{c}2329 \cdot 22 \\
2321 \cdot 14 \\
2312 \cdot 83 \\
2288 \cdot 01 \\
\\
\left\{\begin{array}{l}2264 \cdot 65 \\
(\text { Mitte v. } \\
2264 \cdot 88 \\
\text { und } \\
2264 \cdot 42)\end{array}\right.\end{array}$ & $\left.\begin{array}{c}2328 \cdot 83 \\
2320 \cdot 75 \\
2312 \cdot 45 \\
\\
2287 \cdot 63 \\
\\
\\
\\
2264 \cdot 27 \\
(\text { Mitte v. } \\
2264 \cdot 50 \\
\text { und } \\
2264 \cdot 04)\end{array}\right\}$ & $\begin{array}{l}2299 \cdot 6 \\
2294 \cdot 4 \\
2293 \cdot 9 \\
2291 \cdot 1 \\
\\
2286 \cdot 7 \\
2278 \cdot 4 \\
2276 \cdot 3 \\
2265 \cdot 5\end{array}$ & \begin{tabular}{|}
$2299 \cdot 22$ \\
$\mathbf{2 2 9 4} \cdot \mathbf{0 2}$ \\
$2293 \cdot 52$ \\
$2290 \cdot 72$ \\
\\
$2286 \cdot 32$ \\
$2278 \cdot 02$ \\
$2275 \cdot 92$ \\
$2265 \cdot 12$
\end{tabular} & $\left\{\begin{array}{c}2264 \cdot 77 \\
\text { Mitte von } \\
2264 \cdot 91 \\
\text { und } \\
2264 \cdot 64)\end{array}\right\}$ & $\begin{array}{l}2263 \cdot 6 \\
\\
\mathbf{2 2 4 6} \cdot \mathbf{6} \\
2242 \cdot 2\end{array}$ & $\begin{array}{l}2329 \cdot 5 \\
2321 \cdot 6 \\
2313 \cdot 6\end{array}$ & $\begin{array}{l}2300 \cdot 5 \\
\mathbf{2 2 9 5} \cdot 0 \\
2294 \cdot 6 \\
2291 \cdot 4 \\
2286 \cdot 7 \\
2279 \cdot 6 \\
2277 \cdot 0 \\
2265 \cdot 8\end{array}$ \\
\hline
\end{tabular}

Wittelwerth aus $\{2320 \cdot 92$ erhalten mittelst eines Rowlan d'schen Gitters.

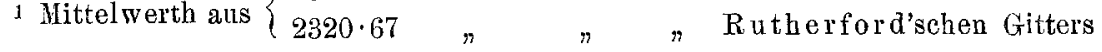
2 Mittelwerth aus $\left\{\begin{array}{l}2312 \cdot 75 \text { erhalten mittelst eines } R \text { owl and'schen Gitters. } \\ 2312 \cdot 66 " n \Rightarrow \text { Rutherford'schen Gitters. }\end{array}\right.$ $\mathrm{n}=$ neblig. 
Spuren eines neuen Elementes.

Tabelle I. (Fortsetzung.)

\begin{tabular}{|c|c|c|c|c|c|c|c|}
\hline \multicolumn{2}{|c|}{ L. Bell } & \multicolumn{2}{|c|}{$\begin{array}{l}\text { Trowbridge } \\
\text { und Sabine }\end{array}$} & \multirow{2}{*}{\multicolumn{2}{|c|}{$\frac{\begin{array}{c}\text { Liveing und } \\
\text { Dewar }\end{array}}{\text { Angström's Scala }}$}} & \multirow{2}{*}{\multicolumn{2}{|c|}{$\begin{array}{c}\begin{array}{c}\text { Hartley und } \\
\text { Adeney }\end{array} \\
\text { Angström's Scala }\end{array}$}} \\
\hline $\begin{array}{c}\text { Rowl. } \\
\text { Seala }\end{array}$ & $\begin{array}{c}\text { Angst. } \\
\text { Seala }\end{array}$ & $\begin{array}{l}\text { Rowl. } \\
\text { Seala }\end{array}$ & $\mid \begin{array}{c}\text { Angst. } \\
\text { Scala }\end{array}$ & & & & \\
\hline \multicolumn{2}{|c|}{$\mathrm{Kadminm}$} & \multicolumn{2}{|c|}{ Kupfer } & $\mathrm{Kadm}$ inm & $\mathrm{K}$ up fer & Kadmium & Kupfer \\
\hline $2193 \cdot 98$ & $2193 \cdot 62$ & $\begin{array}{l}2231 \cdot 0 \\
2230 \cdot 1 \\
2228 \cdot 9 \\
2227 \cdot 8 \\
2226 \cdot 9 \\
2225 \cdot 7 \\
2224 \cdot 8 \\
2218 \cdot 2 \\
2215 \cdot 3 \mathrm{n} \\
2214 \cdot 4 \\
2213 \cdot 0 \\
2210 \cdot 3 \\
2200 \cdot 6 \\
2199 \cdot 8 \mathrm{n} \\
2196 \cdot 9\end{array}$ & $\begin{array}{l}2230 \cdot 63 \\
2229 \cdot 73 \\
2228 \cdot 53 \\
2227 \cdot 43 \\
2226 \cdot 53 \\
2225 \cdot 33 \\
2224 \cdot 43 \\
2217 \cdot 83 \\
2214 \cdot 93 \\
2214 \cdot 03 \\
2212 \cdot 63 \\
2209 \cdot 93 \\
2200 \cdot 23 \\
2199 \cdot 44 \\
2196 \cdot 54 \\
\\
2192 \cdot 04 \\
2189 \cdot 54 \\
2181 \cdot 44 \\
2179 \cdot 14 \\
2174 \cdot 84 \\
2148 \cdot 84 \\
\\
2135 \cdot 75 \\
2134 \cdot 25 \\
2125 \cdot 85 \\
2124 \cdot 95 \\
2122.75 \\
2117 \cdot 15 \\
2111 \cdot 85 \\
2104 \cdot 55\end{array}$ & $\left\{\begin{array}{|l}\left\{\begin{array}{l}2194 \cdot 18 \\
(\text { Mittel })\end{array}\right\} 1 \\
\{\end{array}\right.$ & $\begin{array}{r}2191 \cdot 8 \\
2189 \cdot 2 \\
2178 \cdot 8 \\
2148 \cdot 9 \\
2135 \cdot 7\end{array}$ & $2196 \cdot 4$ & $\begin{array}{l}2232 \cdot 2 \\
2231 \cdot 2 \\
2230 \cdot 0 \\
2229 \cdot 1 \\
2228 \cdot 1 \\
2227 \cdot 0 \\
2226 \cdot 0 \\
2219 \cdot 3 \\
2216 \cdot 5 \\
2215 \cdot 8 \\
2214 \cdot 1 \\
2211 \cdot 3 \\
2200 \cdot 3 \\
2199 \cdot 8 \\
2196 \cdot 5 \\
\\
2192 \cdot 0 \\
2189 \cdot 6 \\
2181 \cdot 0 \\
2179 \cdot 0 \\
2174 \cdot 5 \\
2143 \cdot 8 \\
2135 \cdot 8 \\
2134 \cdot 2 \\
2124 \cdot 4 \\
2124 \cdot 0 \\
2122 \cdot 1 \\
2116 \cdot 0 \\
2110 \cdot 5 \\
2103 \cdot 0\end{array}$ \\
\hline
\end{tabular}

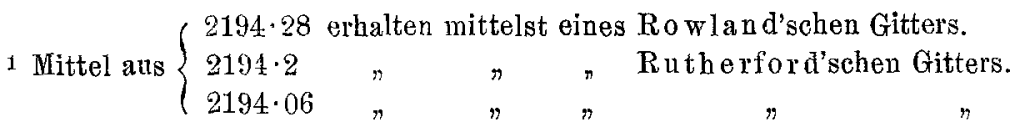


A. Gr î̉ $n \in$ a $\}$ d,

Tabelle II. (Siehe Seite 15.)

\begin{tabular}{|c|c|c|c|c|}
\hline $\begin{array}{c}\text { Tellur, } \\
\text { beobachtet } \\
\text { von Hartley } \\
\text { und Adeney }\end{array}$ & $\mid \begin{array}{c}\text { Antimon, } \\
\text { beobachtet } \\
\text { von Hartley } \\
\text { und Adeney }\end{array}$ & $\begin{array}{c}\text { Kupfer, } \\
\text { beobachtet ron } \\
\text { Hartley und } \\
\text { Adeney }\end{array}$ & $\begin{array}{c}\text { Mittelwerthe } \\
\text { der beobach- } \\
\text { teten Wellen- } \\
\text { längen }\end{array}$ & $\begin{array}{c}\text { Adoptirte } \\
\text { Wellenlängen } \\
\lambda=\begin{array}{c}\text { (Angstr. } \\
\text { Scala) }\end{array}\end{array}$ \\
\hline $3771 \cdot 0$ & $3771 \cdot 0$ & & $3271 \cdot 0$ & $3771 \cdot 0$ \\
\hline $\mathbf{3 5 2 0} \cdot 3$ st. & $3520 \cdot 3$ z. st. & & $3520 \cdot 3$ & $3520 \cdot 3$ \\
\hline 3382. 4 s. st. & $382 \cdot 0$ & & $3382 \cdot 2$ & $3382 \cdot 2$ \\
\hline $3280 \cdot 0$ & $3279 \cdot 7$ & $3280 \cdot 1$ & $3279 \cdot 93$ & $3279 \cdot 93($ auch Ca $)$ \\
\hline 3273. 4 s. st. & $3273 \cdot 0$ & $3273 \cdot 1$ s. st. & $3273 \cdot 2$ & $\left(3273^{2} \quad(\operatorname{archcu})\right.$ \\
\hline $3246 \cdot 8$ s. st. & $3246 \cdot 6$ & 4246.9 z. st. & $3246 \cdot 77$ & $3246 \cdot 77($ auch Cu $)$ \\
\hline $2877 \cdot 4$ & $2877 \cdot 1$ st. & $2877 \cdot 4$ & $2877 \cdot 3$ & $2877 \cdot 3 \quad(\operatorname{auch} \mathrm{Cu})$ \\
\hline $2768 \cdot 6$ z. st. & 2768.9 st. & $2769 \cdot 1$ st. & $2768 \cdot 87$ & $2768 \cdot 87(\operatorname{auch} \mathrm{Cu})$ \\
\hline $2718 \cdot 0$ & $2717 \cdot 9$ st. & $2718 \cdot 4 ?$ & $2718 \cdot 1$ & $2718 \cdot 1 \quad(\operatorname{auch} C u)$ \\
\hline $2702 \cdot 3$ & $2702 \cdot 6$ & $2702 \cdot 7$ st. & $2702 \cdot 53$ & $2702 \cdot 53$ (auch Cu) \\
\hline $2700 \cdot 3$ & $2700 \cdot 2$ & $2700 \cdot 5$ st. & $2700 \cdot 33$ & $2700 \cdot 33($ auch Cu$)$ \\
\hline $2613 \cdot 7$ & $2613 \cdot 7$ & & $2613 \cdot 7$ & $2613 \cdot 7$ \\
\hline $2611 \cdot 3$ & 2611 . 3 st. & & $2611 \cdot 3$ & $2611 \cdot 3$ \\
\hline $2549 \cdot 7 \mathrm{n}$ & $2549 \cdot 8$ & & $2549 \cdot 75$ & $20549 \cdot 75$ \\
\hline $2485 \cdot 3 \mathrm{n}$ & $2485 \cdot 7$ & $2485 \cdot 6$ st. & $2485 \cdot 53$ & $2485 \cdot 53$ (auch Cu) \\
\hline $2479 \cdot 9 \mathrm{n}$ & $2479 \cdot 4$ & & $2479 \cdot 5$ & $2479 \cdot 5$ \\
\hline $2473 \cdot 2$ z. st. & $2473 \cdot 4$ & $2473 \cdot 2$ z. st. & $2473 \cdot 27$ & $2473 \cdot 27$ (auch Cu) \\
\hline $2462 \cdot 0 \mathrm{n}$ & $2462 \cdot 0 \mathrm{n}$. & & $2462 \cdot 0$ & $2462 \cdot 0$ \\
\hline 2438.0 st. & $2438 \cdot 0$ & & $2438 \cdot 0$ & $2438 \cdot 0$ \\
\hline$\left\{\begin{array}{r}2403 \cdot 7 \text { z. st. } \\
\text { breit }\end{array}\right\}$ & $2403 \cdot 8$ & & $2403 \cdot 75$ & $2403 \cdot 75$ \\
\hline $2400 \cdot 0$ z. st. & $2399 \cdot 9$ & $2400 \cdot 1$ st. & $2400 \cdot 0$ & $2400 \cdot 0 \quad(\operatorname{auch~Cu})$ \\
\hline
\end{tabular}


Tabelle II. (Fortsetzung.)

\begin{tabular}{|c|c|c|c|c|}
\hline $\begin{array}{c}\text { Tellur, } \\
\text { beobachtet } \\
\text { von Hartley } \\
\text { und Adeney }\end{array}$ & $\begin{array}{c}\text { Antimon, } \\
\text { beobachtet } \\
\text { von Hartley } \\
\text { und Adeney }\end{array}$ & $\begin{array}{c}\text { Kupfer, } \\
\text { beobachtet von } \\
\text { Hartley.und } \\
\text { Adeney }\end{array}$ & $\begin{array}{c}\text { Mittelwerthe } \\
\text { der beobach- } \\
\text { teten Wellen- } \\
\text { längen }\end{array}$ & $\begin{array}{c}\text { Rectificirte und } \\
\text { adoptirte Wellen- } \\
\text { längen } \lambda= \\
\text { (Angstr. Soala) }\end{array}$ \\
\hline $2870 \cdot 3$ st. & $2370 \cdot 0$ st. & $2870 \cdot 1$ s. st. & $2370 \cdot 13$ & $2369 \cdot 5 \quad$ (auch $\mathrm{Cu})$ \\
\hline $2332 \cdot 0$ st. & $2331 \cdot 8 \mathrm{n}$ & . & $2331 \cdot 9$ & $2331 \cdot 21$ \\
\hline $3225 \cdot 5$ st. & $2325 \cdot 3 \mathrm{n}$. & & $2325 \cdot 4$ & $2324 \cdot 64$ \\
\hline $2288 \cdot 6$ n., br. & 2388.8 z. st. & & $2288 \cdot 7$ & $2287 \cdot 42$ \\
\hline $2280 \cdot 6$ n..z st. & $2280 \cdot 8$ & & $2280 \cdot 7$ & $2279 \cdot 32$ \\
\hline $2277 \cdot 2$ z.st.,n. & $2277 \cdot 1$ & 9277.0 st. & $2277 \cdot 1$ & $2275 \cdot 92($ auch $\mathrm{Cu})$ \\
\hline $2248 \cdot 0$ z. st. & $2248 \cdot 0$ st. & $2248 \cdot 2$ s. st. & $2248 \cdot 07$ & $2246 \cdot 63(\operatorname{auch} \mathrm{Cu})$ \\
\hline $2243 \cdot 3$ z. st. & $2943 \cdot 5$ st. & $2243 \cdot 5$ st.? & $2243 \cdot 43$ & $2241 \cdot 76(\mathrm{a} . \mathrm{Cu}) ?$ \\
\hline $2231 \cdot 3 \mathrm{n}$ & $22831 \cdot 3$ & $2231 \cdot 2$ & $2231 \cdot 27$ & $2229 \cdot 73(\operatorname{auch} \mathrm{Cu})$ \\
\hline $2230 \cdot 3 \mathrm{n}$ & $2330 \cdot 3$ & $2230 \cdot 0$ & $2230 \cdot 2$ & $2228 \cdot 53($ auch $\mathrm{Cu})$ \\
\hline $2229 \cdot 0 \mathrm{n}$ & $22229 \cdot 0$ & $2229 \cdot 1$ & $2229 \cdot 03$ & $2227 \cdot 43(\operatorname{anch} \mathrm{Cu})$ \\
\hline $2223 \cdot 2$ n., br. & $2223 \cdot 5$ & & $2223 \cdot 35$ & $2221 \cdot 83$ \\
\hline $2216 \cdot 0$ n. ${ }^{1}$ & $2216 \cdot 3$ n. 1 & $\left\{\begin{array}{l}2216 \cdot 5 \\
2215 \cdot 8\} \\
2216 \cdot 15 \\
\text { Mitte }\end{array}\right.$ & $2216 \cdot 151$ & $\left\{\begin{array}{l}2214 \cdot 93(\text { auch Cu) } \\
2214.03(\text { auch Cu})\end{array}\right.$ \\
\hline $2211 \cdot 2$ z. st.n. & 22113 & $2211 \cdot 3$ & $2211 \cdot 27$ & $2209 \cdot 93(\operatorname{auch} \mathrm{Cu})$ \\
\hline $2200 \cdot 1 \mathrm{n}$ & $2200 \cdot 3$ & $2200 \cdot 3$ & $2200 \cdot 23$ & $2200 \cdot 23(\operatorname{auch} \mathrm{Cu})$ \\
\hline $2189 \cdot 7$ n. br. & $2189 \cdot 3$ & $2189 \cdot 6 \mathrm{n}$ & $2189 \cdot 53$ & $2189 \cdot 53(\operatorname{auch} \mathrm{Cu})$ \\
\hline $\left.\mid \begin{array}{r}2179 \cdot 2 \text { z, st., } \\
\text { n., br. }\}\end{array}\right\}$ & 2179.9 st. br. & $2179 \cdot 0$ z. st. & $2179 \cdot 07$ & $2179 \cdot 14(\operatorname{anch} \mathrm{Cu})$ \\
\hline $2159 \cdot 7 \mathrm{n.}$ & $2159 \cdot 4$ & & $2159 \cdot 55$ & 215971 \\
\hline $2122 \cdot 5 \mathrm{n}$ & $2122 \cdot 5$ & $2122 \cdot 1 ?$ & $2122 \cdot 37$ & $2122 \cdot 75(\operatorname{auch~Cu})$ \\
\hline $2110 \cdot 5 \mathrm{n}$ & $2110 \cdot 4$ & $2110 \cdot 5$ & $2110 \cdot 47$ & $2111 \cdot 85($ auch $\mathrm{Cu})$ \\
\hline $2050 \cdot 8$ n., br. & $2050 \cdot 5$ & & $2050 \cdot 65$ & $2053 \cdot 59 ?^{2}$ \\
\hline
\end{tabular}


A. Grünwald,

Tabelle III. (Siehe Seite 1\%)

Charakteristische Gruppe (I) übereinstimmender Strahlen des Tellur's und Antimons, eventuell auch des Kupfers.

\begin{tabular}{|c|c|c|c|}
\hline $\begin{array}{c}\text { Adoptirte } \\
\text { Wellenlängen } \\
\text { (siehe Tabelle II, } \\
5 . \text { Colonne!) } \\
\lambda=\text { Angst. Scala }\end{array}$ & $\begin{array}{c}\text { Wasser- } \\
\text { spectrum } \\
\left(\mathrm{H}_{2} \mathrm{O}, \mathrm{H}, b\right) \\
\frac{11}{10} \lambda=\lambda^{\prime}= \\
\text { Angst. Scala }\end{array}$ & $\begin{array}{c}\text { Wasserspectrum, } \\
\text { beobachtet von } \\
\text { G. D. Liveing und } \\
\text { J. Dewar } \\
\text { Angst. Scala }\end{array}$ & $\begin{array}{l}\text { Hydrogen } \\
\text { Virtuelle } \\
\text { Strahlen }(H, b) \\
\frac{4}{5} \lambda^{\prime}=\lambda^{\prime \prime}= \\
\text { Angst Scala }\end{array}$ \\
\hline $2768 \cdot 87($ auch $\mathrm{Cu})$ & $3045 \cdot 76$ & $\left(\mathrm{Huggins} 3046^{\circ} 0\right)$ & $3807 \cdot 20$ \\
\hline $2702 \cdot 53($ anch $\mathrm{Cu})$ & $2972 \cdot 78$ & $\left.\begin{array}{c}\text { Report of } \\
\text { the Brit. } \\
\text { Ass. } 1886\end{array}\right\} 2972 \cdot 8$ & $3715 \cdot 97$ \\
\hline $2700 \cdot 33($ auch $\mathrm{Cu})$ & $2970 \cdot 36$ & $\left\{\begin{array}{l}2970 \cdot 0 \\
\end{array}\right.$ & $3712 \cdot 95$ \\
\hline $2613 \cdot 7$ & $2875 \cdot 07$ & $2875 \cdot 1$ & $3593 \cdot 84$ \\
\hline$? 2485 \cdot 53($ auch $\mathrm{Cu})$ & $2734 \cdot 08$ & $2734 \cdot 3$ & $? 3417 \cdot 60$ \\
\hline $2438 \cdot 0$ & $2681 \cdot 80$ & $2681 \cdot 8$ & $3352 \cdot 25$ \\
\hline $2403 \cdot 75$ & $2644 \cdot 12$ & $2644 \cdot 2$ & $3305 \cdot 15$ \\
\hline
\end{tabular}


Tabelle III. (Fortsetzung.)

\begin{tabular}{|c|c|c|c|}
\hline $\begin{array}{l}\text { Rectificirte und } \\
\text { adoptirte } \\
\text { Wellenlängen } \\
\text { (siehe Tabelle II, } \\
5 . \text { Colonne!) } \\
\lambda=\text { (Angst. Scala }\end{array}$ & $\begin{array}{c}\text { Wasser- } \\
\text { spectrum } \\
\left(H_{2} 0, \text { H. } b\right) \\
\frac{11}{10} \lambda=\lambda^{\prime}= \\
\text { Angst. Scala }\end{array}$ & $\begin{array}{c}\text { Wasserspectrum, } \\
\text { beobachtet von } \\
\text { G. D. Liveing und } \\
\text { J. Dewar } \\
\text { Angst. Scala }\end{array}$ & $\begin{array}{l}\text { Hydrogen } \\
\text { Virtuelle } \\
\text { Strahlen }(\mathrm{H}, b) \\
\frac{5}{4} \lambda^{\prime}=\lambda^{\prime \prime}= \\
\text { Angst. Scala }\end{array}$ \\
\hline $2469 \cdot 5 \quad$ (auch $\mathrm{Cu})$ & $2606 \cdot 45$ & ${ }^{1} \frac{\mathrm{H}^{\prime}}{2}=2606 \cdot 8$ & $3258 \cdot 06$ \\
\hline $2779 \cdot 32$ & $2507 \cdot 25$ & $\left\{\frac{\mathrm{H}^{\prime}}{2}=2507 \cdot 06\right.$ & $3134 \cdot 06$ \\
\hline ?2341-76 (auch Cu?) & $2465 \cdot 93$ & $\left\{\begin{array}{c}2565 \cdot 9 \\
\frac{H^{\prime}}{2}=2465 \cdot 75\end{array}\right.$ & $? 3082 \cdot 41$ \\
\hline $2229 \cdot 73$ (anch $\mathrm{Cu}$ ) & $2452 \cdot 70$ & $\frac{\mathrm{H}^{\prime}}{2}=2452 \cdot 75$ & $3065 \cdot 88$ \\
\hline $2227 \cdot 43($ auch $\mathrm{Cu})$ & $2450 \cdot 17$ & $\frac{\mathrm{H}^{\prime}}{2}=2450 \cdot 12$ & 306271 \\
\hline $2221 \cdot 83$ & $2414 \cdot 01$ & $\frac{\mathrm{H}^{\prime}}{2}=2443 \cdot 84$ & $3055 \cdot 01$ \\
\hline$\{2214 \cdot 93($ anch $\mathrm{Cu})\}$ & $(2436 \cdot 42$ & $\int \frac{\mathrm{H}^{\prime}}{2}=2436 \cdot 2$ & $(3045 \cdot 53$ \\
\hline$(2214 \cdot 03(\operatorname{auch} \mathrm{Cu}))$ & $2435 \cdot 43$ & $2435 \cdot 9$ & $3044 \cdot 25$ \\
\hline $2159 \cdot 71$ & $2375 \cdot 68$ & $2375 \cdot 5$ & $2969 \cdot 60$ \\
\hline
\end{tabular}

- Das Symbol $\frac{\mathrm{H}^{\prime}}{2}$ bedeutet hier und in den folgenden Tabellen halbe Wellenlängen des sogenannnten zusammengesetzten Wasserstoffspectrums; von welchen sich nachweisen lässt, dass sie dem Wasserspectrum angehören. Dieselben müssen in solchen Fällen zu den Vergleichungen herangezogen werden, in welchen die entsprechenden Strahlen des Wasserdampfes zu schwach sind, um auf die photographische Platte zu wirken und messbare Linien zu erzengen. 
Tabelle II a. (Siehe Seite 1.7.)

\begin{tabular}{|c|c|c|c|}
\hline $\begin{array}{c}\text { Hydrogen } \\
\text { Virtuelle } \\
\text { Strahlen }(\mathrm{H}, b) \\
\lambda^{\prime \prime}=\text { Angst. } \\
\text { Scala } \\
\text { (siehe Tab. III, } \\
\text { 4. Colonne!) }\end{array}$ & $\begin{array}{c}\text { Oxygen } \\
\text { Virtuelle } \\
\text { Strahlen } \\
\left(0,0^{\prime}, b\right) \\
\frac{46}{41} \lambda^{\prime \prime}= \\
\text { Angst. Sc, }\end{array}$ & $\mid \begin{array}{c}\text { W asserspectrum } \\
\left(\mathrm{H}_{2} \mathrm{O}, 0, \mathrm{O}^{\prime}, b\right) \\
\frac{23}{32} \cdot \frac{46}{41} \lambda^{\prime \prime}= \\
\text { A ngst. Scala }\end{array}$ & $\begin{array}{c}\text { Wasserspectrum, } \\
\text { beobachtet von } \\
\text { G. D. Liveing und } \\
\text { J. Dewar } \\
\text { Angst. Scala }\end{array}$ \\
\hline $3807 \cdot 20$ & $4271 \cdot 48$ & $3070 \cdot 13$ & $\begin{array}{r}3070 \cdot 0 \\
2996.6\end{array}$ \\
\hline $3715 \cdot 97$ & $4169 \cdot 13$ & $2996 \cdot 56$ & $\frac{H^{\prime}}{2}=2996 \cdot 87$ \\
\hline $3712 \cdot 95$ & $4165 \cdot 74$ & $2994 \cdot 12$ & $\frac{\mathrm{H}^{\prime}}{2}=2994 \cdot 21$ \\
\hline $3593 \cdot 84$ & $4032 \cdot 11$ & $2898 \cdot 07$ & $2898 \cdot 1$ \\
\hline $3417 \cdot 60 ?$ & $3834 \cdot 38$ & $2755 \cdot 95$ & \\
\hline $3352 \cdot 95$ & $3761 \cdot 06$ & $2703 \cdot 25$ & $\frac{\mathrm{H}^{\prime}}{2}=2703 \cdot 13$ \\
\hline $3305 \cdot 15$ & $3708 \cdot 21$ & $2665 \cdot 27$ & $\frac{\mathrm{B}^{\prime}}{2}=2665 \cdot 52$ \\
\hline $3258 \cdot 06$ & $3655 \cdot 38$ & $2627 \cdot 30$ & $2627 \cdot 2$ \\
\hline $3134 \cdot 06$ & $3516 \cdot 26$ & $2527 \cdot 31$ & $\frac{\mathrm{H}^{\prime}}{2}=2527 \cdot 11$ \\
\hline $3082 \cdot 41 ?$ & $3458.31 ?$ & $2485 \cdot 66 ?$ & $2485 \cdot 8$ \\
\hline $3065 \cdot 88$ & $3439 \cdot 76$ & $2472 \cdot 32$ & $\left\{\begin{array}{r}\frac{\mathrm{H}^{\prime}}{2}=2472 \cdot 10 \\
2471 \cdot 9\end{array}\right.$ \\
\hline $3062 \cdot 71$ & $3436 \cdot 21$ & $2469 \cdot 77$ & $2469 \cdot 6$ \\
\hline 3055.01 & $3427 \quad 57$ & $2463 \cdot 56$ & $\frac{\mathrm{H}^{\prime}}{2}=2463 \cdot 97$ \\
\hline$\{3045 \cdot 53$ & $\{3416 \cdot 93$ & $\{2455 \cdot 92$ & $2456 \cdot 0$ \\
\hline$\{3044 \cdot 29$ & $3415 \cdot 54$ & $(2454 \cdot 92$ & $\{2454 \cdot 7$ \\
\hline $2969 \cdot 60$ & $3831 \cdot 74$ & $2394 \cdot 69$ & $\left\{\begin{array}{c}2394 \cdot 8 \\
\frac{\mathrm{H}^{\prime}}{2}=2394 \cdot 96\end{array}\right.$ \\
\hline
\end{tabular}


Tabelle IIIb. (Siehe Seite 17.)

\begin{tabular}{|c|c|c|c|}
\hline $\begin{array}{c}\text { Hydrogen } \\
\text { Virtuelle } \\
\text { Strahlen }(\mathrm{H}, b) \\
\lambda^{\prime \prime}=\text { Angs t. } \\
\text { Scala } \\
\text { (siehe Tab. III, } \\
\text { 4. Colonne!) }\end{array}$ & $\begin{array}{c}\text { Oxygen } \\
\text { Virtuelle } \\
\text { Strahlen } \\
\left(0,0^{\prime}, 0^{\prime \prime}, b\right) \\
\frac{70}{59} \lambda^{\prime \prime}= \\
\text { Angst. Sc. }\end{array}$ & $\begin{array}{c}\text { Wasserspectrum. } \\
\left(\mathbf{H}_{2} \mathrm{O}, 0,0^{\prime}, 0^{\prime \prime}, b\right) \\
\frac{21}{32} \cdot \frac{70}{59} \lambda^{\prime \prime}= \\
\text { Angst. Seala }\end{array}$ & $\begin{array}{l}\text { Wasserspectrum, } \\
\text { beobachtet von } \\
\text { G. D. Liveing und } \\
\text { J. Dewar } \\
\text { Angst. Seala }\end{array}$ \\
\hline $3807 \cdot 20$ & $4517 \cdot 01$ & $2964 \cdot 29$ & $\begin{array}{c}\text { Report of } \\
\text { the Brit. }\left\{2964^{\circ} 5^{1}\right. \\
\text { Ass. } 1886\}^{2893.5}\end{array}$ \\
\hline $3715 \cdot 97$ & $44.08 \cdot 77$ & $2893 \cdot 26$ & $\left\{\frac{H^{\prime}}{2}=2893 \cdot 1\right.$ \\
\hline $3712 \cdot 95$ & $4405 \cdot 19$ & $2890 \cdot 91$ & $2890 \cdot 8$ \\
\hline $3593 \cdot 84$ & $4263 \cdot 87$ & $2798 \cdot 17$ & $\frac{\mathrm{H}^{\prime}}{2}=2797 \cdot 82$ \\
\hline $3417 \cdot 60 ?$ & $4054 \cdot 78$ & $2660 \cdot 95$ & $\begin{array}{r}2660 \cdot 9) \\
2609 \cdot 7\end{array}$ \\
\hline $3352 \cdot 25$ & $3977 \cdot 24$ & $2610 \cdot 06$ & $\frac{\mathrm{H}^{\prime}}{2}=2609 \cdot 86$ \\
\hline $3305 \cdot 15$ & $3921 \cdot 36$ & $2573 \cdot 39$ & $2573 \cdot 4$ \\
\hline $3258 \cdot 06$ & $3865 \cdot 49$ & $2536 \cdot 73$ & $2536 \cdot 6$ \\
\hline $3134 \cdot 06$ & $3718 \cdot 37$ & $2440 \cdot 18$ & $2440 \cdot 3$ \\
\hline $3082 \cdot 41 ?$ & $3657 \cdot 09 ?$ & $2399 \cdot 97 ?$ & $2399 \cdot 4$ \\
\hline $3065 \cdot 88$ & $3637 \cdot 48$ & $2387 \cdot 10$ & $2387 \cdot 0$ \\
\hline $3062 \cdot 71$ & $3633 \cdot 72$ & $2384 \cdot 63$ & $\left\{\begin{array}{r}\frac{\mathrm{H}^{\prime}}{2}=2384 \cdot 78 \\
2384 \cdot 3\end{array}\right.$ \\
\hline $3055 \cdot 01$ & $3624 \cdot 59$ & 237863 & $2378 \cdot 6$ \\
\hline$(3045 \cdot 53$ & $(3613 \cdot 34$ & $(2371 \cdot 25$ & $\int^{\prime} 2371 \cdot 2$ \\
\hline$\{3044 \cdot 29$ & $\{3611 \cdot 87$ & $\{2370 \cdot 29$ & $\frac{H^{\prime}}{2}=2370 \cdot 43$ \\
\hline $2969 \cdot 60$ & $3523 \cdot 25$ & $2312 \cdot 13$ & $\frac{\mathrm{H}^{\prime}}{2}=2312 \cdot 17$ \\
\hline
\end{tabular}

1 Doppelt. Die brechbarere Linie des Paares. 


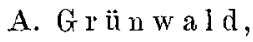

(Gruppe II.)

Tabelle IV. (Siehe Seite 20.)

\begin{tabular}{|c|c|c|c|c|}
\hline $\begin{array}{l}\text { Adoptirte } \\
\text { W ellen- } \\
\text { längen } \\
\text { (siehe Tab. II, } \\
\text { 5. Colonne!) } \\
\text { 2= Angst. } \\
\text { Scala }\end{array}$ & $\begin{array}{c}\text { Wasser- } \\
\text { spectrum } \\
\text { beobachtet von } \\
\text { G. D. Liveing } \\
\text { und J. Dewax } \\
\text { Angst. Scala }\end{array}$ & $\begin{array}{c}\text { Hydrogen } \\
\text { Virtuelle } \\
\text { Strahlen }(\mathrm{H}, a) \\
\frac{3}{2} \lambda=\lambda_{1}= \\
\text { Angst. Scala }\end{array}$ & $\begin{array}{c}\text { Wasser- } \\
\text { dampf } \\
\text { berechnet } \\
\left(\mathrm{H}_{2} \mathrm{O}, \mathrm{H}, a\right) \\
\frac{19}{30} \lambda_{1}= \\
\text { Angst. Se. }\end{array}$ & $\begin{array}{l}\text { Wasserdampf, } \\
\text { beobachtet von } \\
\text { G. D. Liveing } \\
\text { und J. Dewar }\end{array}$ \\
\hline $2613 \cdot 71$ & $2613 \cdot 5$ & $3920 \cdot 55$ & $2483 \cdot 0$ & $\left\{\frac{\mathrm{H}^{\prime}}{2}=2483 \cdot 06\right.$ \\
\hline $2611 \cdot 3$ & $2611 \cdot 0$ & $3916 \cdot 952$ & $2480 \cdot 73$ & $2480 \cdot 7$ \\
\hline $2549 \cdot 75$ & $\frac{\mathrm{H}^{\prime}}{2}=2549 \cdot 5$ & $3824 \cdot 62$ & $2422 \cdot 26$ & $2422 \cdot 4$ \\
\hline $2479 \cdot 5$ & $2479 \cdot 3$ & $3719 \cdot 25$ & $2355 \cdot 52$ & $2355 \cdot 5$ \\
\hline $2462 \cdot 0$ & $\frac{\mathrm{H}^{\prime}}{2}=2162 \cdot 4$ & $3693 \cdot 0$ & $2338 \cdot 9$ & $\frac{\mathrm{H}^{\prime}}{2}=2339^{\circ} 1$ \\
\hline
\end{tabular}

1 Knoten- oder Verzweigungsstrahl dieser mit dex I. Gruppe.

2 Der Hydrogenstrahl 3916.9 als Strahl der Gruppe $(\mathbf{H}, a)$ ist mit Riucksieht auf den der Gruppe $(H, b)$ zugehörigen benachbarten Hydrogenstrahl bei $3916 \cdot 5$ ungefähr (siehe Mathem. Spectralanalyse des Magnesiums und des Kohlenstoffes, Wiener Berichte 1887, S. 61, Tafel Va, 3. Colonne!) besonders bemerkenswerth, indem er dem Strable $\frac{3}{2} \times 3916 \cdot 95=5875 \cdot 42$ (ungefähr) des freien

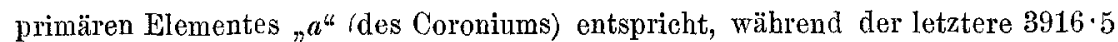
(ungefähr) mit dem Strahle $\frac{3}{2} \times 3916 \cdot 5=5874 \cdot 75$ (ungefähr) des primären Elementes,$b^{\prime \prime}$ dem eigentlichen Heliumstrahl correspondirt. Der Heliumstrahl $\odot D_{3} 5875$ (ungefähr) ist also doppe1t. Die eine minder brechbare Componente gehört dem freien Coronium „a", die andere brechbarere dem Helium, das heisst dein primären Elemente $" b$ " in jener Condensationsform an, in welche es geräth, wenn sich das Hydrogen dissocirt und sich dabei zugleich im Verhältuisse $2: 3$ ausdehnt.

Solche Regionen der Sonnenatmosphäre (oder der Atmosphäre weisser Sterne), in welchen zeitweilig etwa nur die eine der beiden Componenten des Hydrogens vorkommt, werden hiernach nur die eine (entsprechende) der beiden Componenten des Strahles $\odot D_{3}$ emittiren. 
Spuren eines neuen Elementes.

(Zur Gruppe II.) Tabelle IV a. (Siehe Seite 20.)

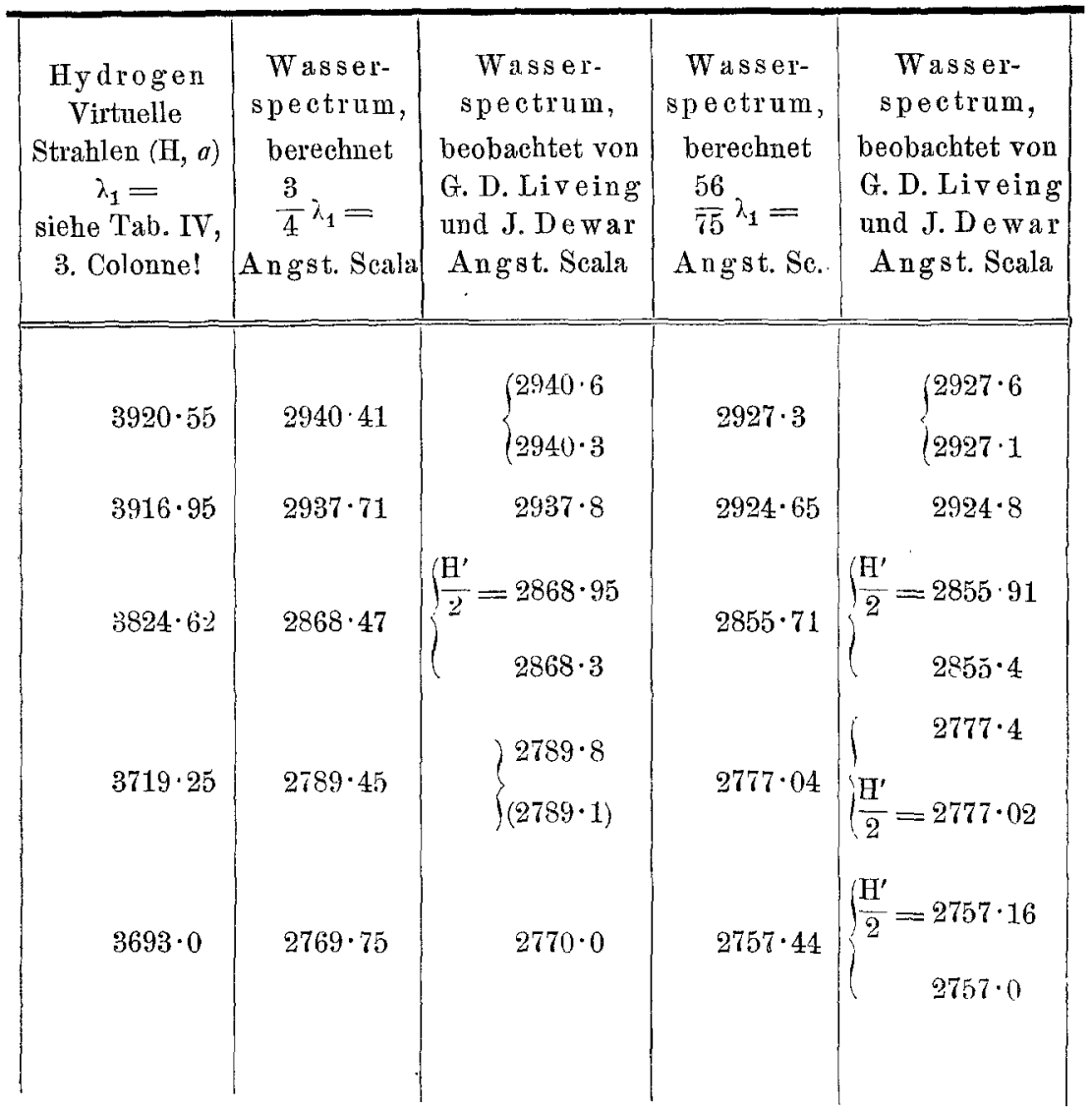

